\title{
On constraint preservation in numerical simulations of Yang-Mills equations
}

\author{
Snorre H. Christiansen, Ragnar Winther ${ }^{\dagger}$
}

October 8, 2004

\begin{abstract}
The problem of constraint preservation for discretizations of non-linear PDEs is addressed on the example of the hyperbolic Yang-Mills equations in temporal gauge. These equations preserve a nonlinear divergence field analogous to the electric charge for Maxwell's equations. We introduce and discuss several discretizations of these equations on finite element spaces of Lie algebra valued differential forms. Numerical experiments indicate that simply restricting the variational formulation to the Galerkin spaces yields substantial drift in the charge, contrary to the linear Maxwell case. We then propose a fully discrete method constrained with Lagrange multipliers, for which we prove discrete charge conservation and observe excellent energy conservation.
\end{abstract}

\section{Introduction}

Many physical phenomena can be accurately described in terms of fields subject to a constrained evolution equation. For instance Maxwell's equations describe an electric field in the vacuum as a divergence-free vector-field satisfying a wave equation. The Navier-Stokes equations describe the velocity field of an incompressible fluid as a divergence-free vector-field subject to a nonlinear evolution equation, involving the pressure which is a Lagrange multiplier for the divergence constraint. For both of these examples, discretization is today relatively well understood, leading to powerful simulation tools.

However for other examples the situation is less satisfactory. In particular, in certain gauges, Einstein's equations of general relativity (GR) reduce to a hyperbolic evolution equation for a Riemannian metric on a space-slice, subject to nonlinear differential constraints, see e.g. Wald [37]. The construction of good discretizations of these equations is an active research topic where much remains to be done. Lehner [24] mentions in particular the need to develop tools to help interpret data from gravitational wave detection experiments, in the new field of "gravitational wave astronomy". It has been suggested that

\footnotetext{
${ }^{*}$ CMA c/o Dept. of Math., University of Oslo, P.O. Box 1053 Blindern, NO-0316 Oslo, Norway. email: snorrec@math.uio.no.

†CMA c/o Dept. of Math., University of Oslo, P.O. Box 1053 Blindern, NO-0316 Oslo, Norway, email: ragnar.winther@cma.uio.no.
} 
the problems encountered for simulating Einstein's equations are linked to difficulties for discretizations to preserve nonlinear differential constraints, since hyperbolicity might be lost outside the constraint manifold.

To gain insight into the generic problem of constraint preservation for nonlinear PDEs, we study in this paper the Yang-Mills equations in their hyperbolic form. These are evolution partial differential equations preserving a nonlinear constraint on the Cauchy data (initial values). However in contrast to the initial value formulation of Einstein's equations, the Yang-Mills system remains stable even if the constraint is violated. The Yang-Mills equations appear relatively ripe for numerical analysis and could therefore serve as a stepping stone toward the successful simulation of more complicated equations. There are several reasons for this belief. First, concerning space discretization, when the Lie algebra is trivial, Yang-Mills equations reduce to Maxwell's equations and the Galerkin discretization of these on edge elements spaces is now relatively well understood (see $\S 3.1$ for references). Second, concerning time discretization, recent years have seen a deeper understanding of geometric properties of time-stepping methods (see also §3.1). These two points are of course not completely distinct as they can be seen as facets of space-time variational principles. A third reason for optimism is that contrary to many other non-linear equations one has analysis tools for studying relatively rough solutions to Yang-Mills equations, perhaps enabling norm estimates on the non-smooth functions that appear in finite element discretizations (see $\S 2.2$ ).

Briefly put, Yang-Mills equations are Euler-Lagrange equations for a time dependent gauge potential, which can be deduced from a Lagrangian which is invariant under a certain group of transformations called gauge transformations. This invariance provides a conservation property similar to (electric) charge conservation for Maxwell's equations. However, the natural discretization space for gauge potentials appears not to be invariant under the natural candidate for a subgroup of gauge transformations, contrary to the linear case of Maxwell's equations. We study numerically the effect of this non-invariance on charge conservation and discuss ways to compensate for it by imposing constraints with Lagrange multipliers.

The paper is organized as follows. A first part is devoted to introducing the equations and their setting. In $\S 2.1$ we define Lie algebra valued differential forms and describe the basic operations on them that we will need. Then, in $\S 2.2$, we introduce the Yang-Mills equations from a variational principle and relate these equations to Maxwell's equations. In $\S 2.3$ we prove the charge conservation property we are interested in. In a second part we turn to discretizations and study first, in $\S 3.1$, the (semi-)discretizations obtained by restricting the Lagrangian to a finite dimensional space of gauge potentials. We show numerical results indicating that charge conservation is bad even though other parameters appear to be good. Motivated by this failure we then, in $\S 3.2$, discuss ways to impose charge conservation actively and show excellent numerical results for one of them. We have also included some remarks pertaining to the implementation of the proposed schemes in $§ 3.3$. Finally we conclude with some indications on possible directions in which to pursue the investigations. 


\section{Yang-Mills equations}

\subsection{Lie algebra valued differential forms}

- In this section we introduce notations and collect some facts about Lie algebra valued differential forms. Short introductions to this topic can be found in Taylor [35] (appendix C) and Donaldson - Kronheimer [12] (chapter 2). Here we restrict attention to what are called trivial principal bundles, so in fact we shall not need the definition of a principal bundle at all. This leads to many simplifications in the presentation. Some of the global topological properties of the theory are thus lost, but this seems unimportant for the purposes of this paper.

- We will define many objects in terms of tensor products. This is convenient not only for the exposition but also for implementing algorithms since tensor products of operators corresponds to Kronecker products of matrices. A handy introduction to tensor products of vector spaces can be found in Ryan [31] (chapter 1). We will come back to issues of implementation later. Many tensor products are canonically isomorphic to spaces of (multi-) linear maps. In particular, if $\mathrm{E}$ denotes a finite-dimensional vector space, the space of $p$-linear forms on $E$ can be identified with the $p$-fold tensor product $\left(\otimes^{p} E^{\star}\right)$. If $u: E^{p} \rightarrow \mathbb{R}$ is a $p$-linear form and $v: E^{q} \rightarrow \mathbb{R}$ is a $q$-linear form, their tensor product $u \otimes v$ is the $(p+q)$-linear form $E^{p+q} \rightarrow \mathbb{R}$ defined by:

$$
(u \otimes v)\left(\xi_{1}, \cdots, \xi_{p+q}\right)=u\left(\xi_{1}, \cdots, \xi_{p}\right) v\left(\xi_{p+1}, \cdots, \xi_{p+q}\right) .
$$

The antisymmetrization operator on the space of $p$-linear forms is the projector $\alpha_{p}$ defined by:

$$
\left(\alpha_{p} u\right)\left(\xi_{1}, \cdots, \xi_{p}\right)=\frac{1}{p !} \sum_{\sigma \in \mathfrak{S}(p)} \epsilon(\sigma) u\left(\xi_{\sigma(1)}, \cdots, \xi_{\sigma(p)}\right) .
$$

where $\mathfrak{S}(p)$ denotes the group of permutations of the set $\{1,2, \cdots, p\}$ and $\epsilon$ is the signature morphism. Antisymmetric $p$-linear forms are also said to be alternating.

- We will use freely the language of (real-valued) differential forms, as exposed for instance in Lang [23], but recall some basic facts. Let $M$ be a finite dimensional real and smooth manifold. The tangent space of $M$ at a point $x$ is denoted $\mathrm{T}_{x} M$. For any integer $p$, a $p$-form on $M$ is an object $u$ which to any point $x \in M$ associates a $p$-linear alternating map $u_{x}:\left(\mathrm{T}_{x} M\right)^{p} \rightarrow \mathbb{R}$. We denote by $\Omega^{p}$ the space of (smooth) $p$-forms on $M$. When $p>\operatorname{dim} M, \Omega^{p}$ is

reduced to $0 . \Omega^{0}$ is the space of real-valued functions on $M$. The direct sum of the spaces $\Omega^{p}$ is denoted $\Omega$. The wedge product is a bilinear associative map $\wedge: \Omega \times \Omega \rightarrow \Omega$ such that for each integers $p$ and $q$ :

$$
\wedge: \Omega^{p} \times \Omega^{q} \rightarrow \Omega^{p+q},
$$

For each $u \in \Omega^{p}$ and $v \in \Omega^{q}$ it is defined at $x \in M$ by:

$$
(u \wedge v)_{x}=\frac{(p+q) !}{p ! q !} \alpha_{p}\left(u_{x} \otimes v_{x}\right)
$$


We then have:

$$
v \wedge u=(-1)^{p q} u \wedge v
$$

The exterior derivative is a linear map $\mathrm{d}: \Omega \rightarrow \Omega$ such that for each integer $p$ :

$$
\mathrm{d}: \Omega^{p} \rightarrow \Omega^{p+1} \text {. }
$$

In charts it can be constructed by antisymmetrizing the full differential. It is also characterized by the following two properties: If $u \in \Omega^{0}$ then for each $x \in M,(\mathrm{~d} u)_{x}=\mathrm{D} u(x): \mathrm{T}_{x} \rightarrow \mathbb{R}$ is the usual differential of $u$ at $x$ (also called derivative or tangent map). Moreover for each $u \in \Omega^{p}$ and $v \in \Omega^{q}$ we have:

$$
\mathrm{d}(u \wedge v)=(\mathrm{d} u) \wedge v+(-1)^{p} u \wedge \mathrm{d} v .
$$

Essentially because the full second-order differential (say in some chart) is symmetric (Schwartz lemma), the operator $d$ satisfies $\mathrm{d}^{2}=0$, making the sequence of spaces $\left(\Omega^{p}\right)$ what is called a complex.

- We now fix a finite dimensional real Lie algebra $\mathfrak{g}$. That is, a finite dimensional real vector space equipped with a bilinear map $[\cdot, \cdot]: \mathfrak{g} \times \mathfrak{g} \rightarrow \mathfrak{g}$ called Lie bracket, which is antisymmetric and satisfies the Jacobi identity. These two last properties are equivalent to the statements that for all $a, b, c \in \mathfrak{g}$ :

$$
\begin{aligned}
0 & =[a, b]+[b, a] \\
{[a,[b, c]] } & =[[a, b], c]+[b,[a, c]] .
\end{aligned}
$$

The last one expresses that for each $a \in \mathfrak{g},[a, \cdot]$ acts as a derivation on $[\cdot, \cdot]$.

- By a $\mathfrak{g}$-valued $p$-form (on $M$ ) we mean an object $A$ which to any point $x \in M$ associates a $p$-linear alternating map $A_{x}:\left(\mathrm{T}_{x} M\right)^{p} \rightarrow \mathfrak{g}$. The space of $\mathfrak{g}$-valued $p$-forms is canonically isomorphic to the tensor product $\Omega^{p} \otimes \mathfrak{g}$. Explicitly, if $u \in \Omega^{p}$ and $a \in \mathfrak{g}, u \otimes a$ can be identified with the $\mathfrak{g}$-valued $p$-form defined at any $x \in M$ by:

$$
\left(\xi_{1}, \cdots, \xi_{p}\right) \mapsto u_{x}\left(\xi_{1}, \cdots, \xi_{p}\right) a,
$$

and any $\mathfrak{g}$-valued $p$-form is a linear combination of (at most $\operatorname{dim} \mathfrak{g}$ ) such forms. The bracket of $\mathfrak{g}$-valued forms is a bilinear operator $(\Omega \otimes \mathfrak{g}) \times(\Omega \otimes \mathfrak{g}) \rightarrow \Omega \otimes \mathfrak{g}$ giving restrictions $\left(\Omega^{p} \otimes \mathfrak{g}\right) \times\left(\Omega^{q} \otimes \mathfrak{g}\right) \rightarrow \Omega^{p+q} \otimes \mathfrak{g}$. It is denoted $[\cdot, \cdot]$ and uniquely characterized by the property that for all $u \in \Omega^{p}, a \in \mathfrak{g}, v \in \Omega^{q}$ and $b \in \mathfrak{g}$ :

$$
[u \otimes a, v \otimes b]=(u \wedge v) \otimes[a, b] .
$$

In other words, we consider the tensor product of the standard wedge $\wedge$ defined on (real-valued) differential forms on $M$, with the Lie bracket $[\cdot, \cdot]$ on $\mathfrak{g}$. This gives $\Omega \otimes \mathfrak{g}$ the structure of a graded Lie algebra. That is, if we have $A \in \Omega^{p} \otimes \mathfrak{g}$, $B \in \Omega^{q} \otimes \mathfrak{g}$ and $C \in \Omega^{r} \otimes \mathfrak{g}$ then we deduce from (2.8), (2.9) and (2.5):

$$
\begin{aligned}
0 & =[A, B]+(-1)^{p q}[B, A], \\
{[A,[B, C]] } & =[[A, B], C]+(-1)^{p q}[B,[A, C]] .
\end{aligned}
$$

The exterior derivative is extended to an operator $\mathrm{d}: \Omega \otimes \mathfrak{g} \rightarrow \Omega \otimes \mathfrak{g}$ inducing maps $\Omega^{p} \otimes \mathfrak{g} \rightarrow \Omega^{p+1} \otimes \mathfrak{g}$. It is uniquely characterized by the property that:

$$
\forall u \in \Omega^{p} \forall a \in \mathfrak{g} \quad \mathrm{d}(u \otimes a)=(\mathrm{d} u) \otimes a .
$$


- If $A$ is a gauge potential i.e. an element of $\Omega^{1} \otimes \mathfrak{g}$ we associate with it a covariant exterior derivative denoted $\mathrm{d}_{A}$ defined by:

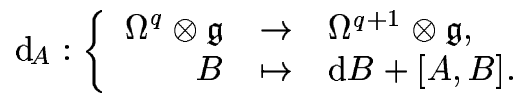

It is a derivation in the sense that if $B \in \Omega^{q} \otimes \mathfrak{g}$ and $C \in \Omega^{r} \otimes \mathfrak{g}$ we have (compare with (2.9) and (2.7)):

$$
\mathrm{d}_{A}[B, C]=\left[\mathrm{d}_{A} B, C\right]+(-1)^{q}\left[B, \mathrm{~d}_{A} C\right] .
$$

The curvature of a gauge potential $A$ is the element $\mathcal{C}(A)$ of $\Omega^{2} \otimes \mathfrak{g}$ defined by Cartan's formula:

$$
\mathcal{C}(A)=\mathrm{d} A+(1 / 2)[A, A] .
$$

This definition is motivated by the property that for all gauge potentials $A$ and all $B \in \Omega^{q} \otimes \mathfrak{g}$ we have:

$$
\mathrm{d}_{A} \mathrm{~d}_{A} B=[\mathcal{C}(A), B] .
$$

We remark that the bilinear map $[\cdot, \cdot]:\left(\Omega^{1} \otimes \mathfrak{g}\right)^{2} \rightarrow \Omega^{2} \otimes \mathfrak{g}$ is symmetric. Therefore the differential DC of the curvature map $\mathcal{C}: \Omega^{1} \otimes \mathfrak{g} \rightarrow \Omega^{2} \otimes \mathfrak{g}$, satisfies for all $A, A^{\prime} \in \Omega^{1} \otimes \mathfrak{g}$ :

$$
\mathrm{DC}(A) A^{\prime}=\mathrm{d}_{A} A^{\prime} .
$$

We also have the Bianchi identity:

$$
\mathrm{d}_{A} \mathcal{C}(A)=0 .
$$

- We suppose that $\mathfrak{g}$ is the Lie algebra associated with a Lie group $G$. For generalities on Lie groups we refer to e.g. Duisterman-Kolk [13]. For notational purposes we suppose that $G$ is a matrix Lie group. Given a function $Q: M \rightarrow G$, the associated gauge transformation $\mathcal{G}_{Q}$ is the map which transforms a gauge potential $A$ into the gauge potential $A^{\prime}=\mathcal{G}_{Q}(A)$ defined by:

$$
A^{\prime}=Q A Q^{-1}-(\mathrm{D} Q) Q^{-1} .
$$

These notations call for some comments. First, for a given $q \in G$, one shows that $a \mapsto q a q^{-1}$ maps $\mathfrak{g}$ into $\mathfrak{g}$ linearly so that the first term on the right hand side of (2.21) can be interpreted pointwise. Second, also for a given $q \in G$, (right and left) multiplication by $q^{-1}$ maps the tangent space $\mathrm{T}_{q} G$ of $G$ at $q$ linearly into $\mathfrak{g}$. And for any function $Q: M \rightarrow G$ the differential of $Q$ gives rise to linear maps $\mathrm{D} Q(x): \mathrm{T}_{x} M \rightarrow \mathrm{T}_{Q(x)} G$. Thus $A^{\prime}$ defined as above is indeed a gauge potential.

Gauge transformations behave nicely with covariant derivatives and curvature. With $A^{\prime}=\mathcal{G}_{Q}(A)$ as before, we have:

$$
\mathrm{d}_{A^{\prime}}\left(Q B Q^{-1}\right)=Q\left(\mathrm{~d}_{A} B\right) Q^{-1},
$$

and:

$$
\mathcal{C}\left(A^{\prime}\right)=Q \mathcal{C}(A) Q^{-1} .
$$

Moreover $\mathcal{G}$ determines a group action in the sense that:

$$
\mathcal{G}_{Q^{\prime}} \circ \mathcal{G}_{Q}=\mathcal{G}_{Q^{\prime} Q},
$$

where on the right hand side we use pointwise multiplication to define the product $Q^{\prime} Q$. 
- We suppose that a Riemannian metric is given on $M$. That is, for any $x \in M$ we have an Euclidean scalar product on $\mathrm{T}_{x} M$, depending smoothly on $x$. It enables us construct $\mathrm{L}^{2}$-products on $\Omega$ as follows. First, for any Euclidean space $E$ (we have in mind the tangent spaces $\mathrm{T}_{x} M$ ), whose scalar product we denote by $(\cdot \mid \cdot)$, we first obtain an Euclidean structure on the dual $E^{\star}$, whose scalar product we also denote by $(\cdot \mid \cdot)$, by requiring that $u \mapsto(u \mid \cdot)$ is an isometry $E \rightarrow E^{\star}$. Next, on the space of $p$-linear forms on $E^{p}$ (not necessarily alternating), there is a unique scalar product such that, for any $u_{1}, \cdots, u_{p}$, $v_{1}, \cdots, v_{p}$ in $E^{\star}$ :

$$
\left(u_{1} \otimes \cdots \otimes u_{p} \mid v_{1} \otimes \cdots \otimes v_{p}\right)=\left(u_{1} \mid v_{1}\right) \cdots\left(u_{p} \mid v_{p}\right) .
$$

This scalar product can be restricted to the subspace of alternating $p$-linear forms on $E^{p}$. This construction provides for each $x \in M$, an Euclidean structure on the space of $p$-linear forms on $\left(\mathrm{T}_{x} M\right)^{p}$, denoted $(\cdot \mid \cdot)_{x}$. We then obtain a scalar product $\langle\cdot, \cdot\rangle$ on $\Omega$ defined by:

$$
\langle u, v\rangle=\int_{M}\left(u_{x} \mid v_{x}\right)_{x} \mathrm{~d} x,
$$

where we integrate using the measure on $M$ provided by the Riemannian metric.

- We suppose furthermore that the Lie group $G$ is compact. Then on $\mathfrak{g}$ there is a scalar product denoted $(\cdot \mid \cdot)$ which is compatible in the sense that for all $q \in G$ the map $a \mapsto q a q^{-1}$ is an isometry of $\mathfrak{g}$ (see e.g. [13] p. 135). This property implies that:

$$
\forall a, b, c \in \mathfrak{g} \quad([a, b] \mid c)+(b \mid[a, c])=0 .
$$

With the help of this scalar product and a Riemannian metric on $M$ we can construct $\mathrm{L}^{2}$ products on $\Omega \otimes \mathfrak{g}$, as well as $\mathrm{L}^{q}$ spaces, by slightly extending the preceding method. Given as before an Euclidean space $E$ one first shows that there is a unique scalar product on $\left(\otimes^{p} E^{\star}\right) \otimes \mathfrak{g}$ such that for $u, v \in \otimes^{p} E^{\star}$ and $a, b \in \mathfrak{g}:$

$$
(u \otimes a \mid v \otimes b)=(u \mid v)(a \mid b) .
$$

This provides scalar products on the spaces $\left(\otimes^{p}\left(\mathrm{~T}_{x} M\right)^{\star}\right) \otimes \mathfrak{g}$ as before, as well as a scalar product on $\Omega \otimes \mathfrak{g}$ denoted $\langle\cdot, \cdot\rangle$. We also obtain $\mathrm{L}^{q}$ norms defined by:

$$
\|A\|_{\mathrm{L}^{q}}=\left(\int_{M}\left|A_{x}\right|_{x}^{q} \mathrm{~d} x\right)^{1 / q},
$$

where $|\cdot|_{x}$ is the Euclidean norm on $\left(\otimes^{p}\left(\mathrm{~T}_{x} M\right)^{\star}\right) \otimes \mathfrak{g}$ defined by the constructed scalar product. At least for our purposes these norms behave like standard $\mathrm{L}^{q}$ norms on scalar functions, for which we refer to Folland [15].

From eq. (2.27) we deduce that for any $A \in \Omega^{0} \otimes \mathfrak{g}$ and any $B, C \in \Omega^{p} \otimes \mathfrak{g}$ we have:

$$
\langle[A, B], C\rangle+\langle B,[A, C]\rangle=0 .
$$

In particular the case $B=C$ yields:

$$
\langle[A, B], B\rangle=0 .
$$




\subsection{Yang-Mills Lagrangian and equations}

- In accordance with notions introduced in the previous section we consider the following setting and notations: $M$ is a compact Riemannian manifold and $G$ is a compact Lie group with associated Lie algebra $\mathfrak{g}$. A compatible scalar product on $\mathfrak{g}$ is chosen. The $\mathrm{L}^{2}$-duality on $\Omega \otimes \mathfrak{g}$ with respect to the Riemannian metric on $M$ and the compatible scalar product on $\mathfrak{g}$ is denoted $\langle\cdot, \cdot\rangle$. The $\mathrm{L}^{2}$ norm is denoted $\|\cdot\|$.

- The hyperbolic Yang-Mills equations, in the temporal gauge, is an equation for gauge potentials on $M$ that can be derived from the Lagrangian $\mathcal{L}$ defined on $\left(\Omega^{1} \otimes \mathfrak{g}\right)^{2}$ by:

$$
\mathcal{L}\left(A, A^{\prime}\right)=(1 / 2)\left\|A^{\prime}\right\|^{2}-(1 / 2)\|\mathcal{C}(A)\|^{2} .
$$

We look for time-dependent gauge potentials $A: t \mapsto A(t) \in \Omega^{1} \otimes \mathfrak{g}$ satisfying the stationary action principle. That is, we look for trajectories $A$ which, for any $t, t^{\prime} \in \mathbb{R}$, are critical points of the action integral:

$$
B \mapsto \int_{t}^{t^{\prime}} \mathcal{L}(B(s), \dot{B}(s)) \mathrm{d} s,
$$

among trajectories $B$ such that $B(t)=A(t)$ and $B\left(t^{\prime}\right)=A\left(t^{\prime}\right)$.

The associated Euler-Lagrange equation for a time-dependent gauge potential $A: t \mapsto A(t)$ is (at any time $t$ ):

$$
\begin{aligned}
\forall A^{\prime} \in \Omega^{1} \otimes \mathfrak{g}\left\langle\ddot{A}, A^{\prime}\right\rangle & =-\left\langle\mathcal{C}(A), \mathrm{DC}(A) A^{\prime}\right\rangle, \\
& =-\left\langle\mathcal{C}(A), \mathrm{d}_{A} A^{\prime}\right\rangle .
\end{aligned}
$$

The so-called strong formulation of this equation is obtained by introducing $\mathrm{d}_{A}^{\star}$, the formal adjoint of $\mathrm{d}_{A}$, with respect to $\mathrm{L}^{2}$-duality on $M$ :

$$
\ddot{A}=-\mathrm{d}_{A}^{\star} \mathcal{C}(A) .
$$

To us the strong formulation is mainly a convenient notation. Discretizations are guided by the weak formulation or indeed the stationary action principle.

- Various first order formulations are useful. With a time-dependent gauge potential $A: t \mapsto A(t)$ we associate time-dependent fields $E: t \mapsto E(t) \in \Omega^{1} \otimes \mathfrak{g}$ and $H: t \mapsto H(t) \in \Omega^{2} \otimes \mathfrak{g}$ defined by:

$$
\begin{aligned}
E(t) & =-\dot{A}(t), \\
H(t) & =\mathcal{C}(A(t)) .
\end{aligned}
$$

We remark that these two equations imply the following evolution equation for $H$ :

$$
\dot{H}(t)=\mathrm{DC}(A(t)) \dot{A}(t)=-\mathrm{d}_{A} E .
$$

So we get (in strong form):

$$
\begin{aligned}
\dot{A} & =-E, \\
\dot{E} & =\mathrm{d}_{A}^{\star} H, \\
\dot{H} & =-\mathrm{d}_{A} E .
\end{aligned}
$$


Conversely, if $(A, E, H)$ satisfies the above system, and the initial condition $H(0)=\mathcal{C}(A(0))$, then for all times $t, H(t)=\mathcal{C}(A(t))$ and $A$ satisfies the Euler Lagrange equation (2.35).

We will also use the following first order formulation involving only the fields $A$ and $E$ :

$$
\begin{aligned}
\dot{A} & =-E, \\
\dot{E} & =\mathrm{d}_{A}^{\star} \mathcal{C}(A) .
\end{aligned}
$$

It differs from the standard Hamiltonian reformulation of Euler-Lagrange equations by the sign appearing in eq. (2.43). The choice of signs is motivated by the conventions used in electromagnetics, to which we shall arrive shortly.

- We have stated most of the above results as if every field was smooth. For the purposes of numerical analysis it it useful however to keep in mind the following existence and uniqueness results for non-smooth solutions of the Yang-Mills equations. Perhaps most importantly to us, Klainerman and Machedon [22] have proved global well-posedness in $\mathrm{H}_{l o c}^{1}$ for initial data with finite energy. An essential ingredient here is a compactness result of Uhlenbeck [36]. Tao [34] has obtained local results for norms weaker than $\mathrm{H}_{l o c}^{1}$. Earlier results include existence and uniqueness for high-order Sobolev norms by Segal [32], see also Eardley-Moncrief [14].

Actually even the $\mathrm{H}_{l o c}^{1}$ framework is somewhat inadequate for numerical analysis. It appears mainly because we have gauge potentials $A$, say in $\mathrm{L}^{2}$, with exterior derivative $\mathrm{d} A$ in $\mathrm{L}^{2}$, for which we have some control over $\mathrm{d}^{\star} A$. Experience from Maxwell's equations (to which we devote the next paragraph) indicates that we should consider discretization spaces consisting of potentials $A \in \mathrm{L}^{2}$ such that $\mathrm{d} A \in \mathrm{L}^{2}$, but for which $\mathrm{d}^{\star} A$ is not necessarily integrable. This term should rather be controlled by the good behavior of $\left\langle A, \mathrm{~d} P^{\prime}\right\rangle$ for a large enough space of "test functions" $P^{\prime} \in \mathrm{L}^{2}$ satisfying $\mathrm{d} P^{\prime} \in \mathrm{L}^{2}$.

The numerical analysis is as of today incomplete and we do not claim to have found the right framework for carrying it out.

- Many of the results of this paper are motivated by analogies with Maxwell's equations and we now explicitate this link. In the context of electromagnetics, $M$ is three-dimensional, $E$ is the electric field and $H$ is the magnetic field. We choose $G$ to be the group:

$$
\mathbb{U}=\{u \in \mathbb{C}:|u|=1\},
$$

so that $\mathfrak{g}$ can be identified with $i \mathbb{R} \subset \mathbb{C}$. Since $\mathbb{U}$ is commutative the Lie bracket is trivial, leading to many simplifications. From eq. $(2.41,2.42)$ we obtain the familiar (vacuum) Maxwell equations for $E$ and $H$, given here in vector notation:

$$
\begin{array}{rlr}
\dot{E} & = & \operatorname{curl} H, \\
\dot{H} & =-\operatorname{curl} E .
\end{array}
$$

Eq. $(2.37,2.38)$ express that the magnetic potential $A$ is a solution of (also in vector notation):

$$
\begin{aligned}
\dot{A} & =-E, \\
\operatorname{curl} A & =H .
\end{aligned}
$$


The strong form of the Euler-Lagrange eq. (2.36) for $A$ is:

$$
\ddot{A}=-\operatorname{curl} \operatorname{curl} A \text {. }
$$

All fields here are $\mathbb{R}$-proportional to the complex number $i$, so it is customary to cancel it out. Other choices of magnetic potentials are often used but in this paper we restrict attention to the above choice of gauge, called temporal gauge (because $A$ can be identified with a 1 -form on time-space $\mathbb{R} \times M$ having no component along the time axis).

Maxwell's equations $(2.46,2.47)$ preserve the following constraints on the initial data:

$$
\begin{aligned}
\operatorname{div} E & =0, \\
\operatorname{div} H & =0 .
\end{aligned}
$$

This follows immediately from the identity div curl $=0$. The Yang-Mills equations have similar constraints as we shall see in the next section.

In this presentation we have of course reversed the historical order, since Yang and Mills obtained their equations in an effort to generalize Maxwell's equations to the case where $G$ is a non-commutative Lie group such as $\mathrm{SU}(2)$.

\subsection{Conservation properties}

- For Maxwell's equations $(2.46,2.47)$ the two divergence constraints $(2.51$, 2.52) appear as completely symmetric. But once a magnetic potential is introduced as in $(2.48,2.49)$, these two constraints acquire rather different meanings. This will become even more apparent for the Yang-Mills equations, which have similar constraints. The generalization to Yang-Mills equations, of the divergence constraint (2.52) on the magnetic field, is the Bianchi identity (2.20). As we shall see, it is not a problem to handle numerically, at least for the discretizations we have chosen. On the other hand the following identity, which is analogous to charge conservation (2.51), is numerically more challenging. We should have the constraint:

$$
\forall P^{\prime} \in \Omega^{0} \otimes \mathfrak{g} \quad\left\langle\dot{A}, \mathrm{~d}_{A} P^{\prime}\right\rangle=0 .
$$

In general (not necessarily temporal) gauge this equation is actually one of the Yang-Mills equations. In our setting however, it appears as a constraint which is preserved by the flow and which should be satisfied initially. We now check this in two (related) ways.

- Pick $P^{\prime} \in \Omega^{0} \otimes \mathfrak{g}$, and regard it as a constant function of time. Putting $A^{\prime}=\mathrm{d}_{A} P^{\prime}$ in eq. (2.35) we obtain (from eq. (2.18) and (2.31)):

$$
\left\langle\ddot{A}, \mathrm{~d}_{A} P^{\prime}\right\rangle=-\left\langle\mathcal{C}(A),\left[\mathcal{C}(A), P^{\prime}\right]\right\rangle=0 .
$$

We also remark that:

$$
\left\langle\dot{A},\left[\dot{A}, P^{\prime}\right]\right\rangle=0 .
$$

It follows that:

$$
\left\langle\dot{A}, \mathrm{~d}_{A} P^{\prime}\right\rangle^{\cdot}=\left\langle\ddot{A}, \mathrm{~d}_{A} P^{\prime}\right\rangle+\left\langle\dot{A},\left[\dot{A}, P^{\prime}\right]\right\rangle=0 .
$$


In analogy with Maxwell's equations we will refer to this conservation property as charge conservation. As already mentioned, condition (2.53) should also be satisfied initially. Thus, with $\dot{A}=-E$, the couple $(A(t), E(t))$ should evolve on the (in general infinite dimensional, non-linear and perhaps singular) "manifold" with equation:

$$
\forall P^{\prime} \in \Omega^{0} \otimes \mathfrak{g} \quad\left\langle E, \mathrm{~d}_{A} P^{\prime}\right\rangle=0 .
$$

- We now show how this property can be obtained using Noether's theorem. First fix a map $Q: M \rightarrow G$, and denote by $\mathcal{F}=\mathcal{G}_{Q}: \Omega^{1} \otimes \mathfrak{g} \rightarrow \Omega^{1} \otimes \mathfrak{g}$ the associated gauge transformation. For all gauge potentials $A, A^{\prime} \in \Omega^{1} \otimes \mathfrak{g}$ we have (using (2.23), and the compatibility of the scalar product):

$$
\begin{aligned}
\mathcal{L}\left(\mathcal{F}(A), \mathrm{D} \mathcal{F}(A) A^{\prime}\right) & =(1 / 2)\left\|Q A^{\prime} Q^{-1}\right\|^{2}-(1 / 2)\left\|Q \mathcal{C}(A) Q^{-1}\right\|^{2} \\
& =\mathcal{L}\left(A, A^{\prime}\right) .
\end{aligned}
$$

In other words the Lagrangian $\mathcal{L}$ is invariant under all gauge transformations $\mathcal{G}_{Q}$ associated with maps $Q: M \rightarrow G$. On the other hand, if we fix a gauge potential $A$ and differentiate the map $Q \mapsto \mathcal{G}_{Q}(A)$ at the constant map $I: M \rightarrow G$ equal to the unit of $G$, we get the following linear map:

$$
\Gamma(A):\left\{\begin{array}{rll}
\Omega^{0} \otimes \mathfrak{g} & \rightarrow \Omega^{1} \otimes \mathfrak{g} \\
P^{\prime} & \mapsto-\mathrm{d}_{A} P^{\prime}
\end{array} .\right.
$$

Therefore, by Noether's theorem, (see e.g. Marsden-West [26] p. 369) for each $P^{\prime} \in \Omega^{0} \otimes \mathfrak{g}$ the following function on $\left(\Omega^{1} \otimes \mathfrak{g}\right)^{2}$ is constant on trajectories $t \mapsto(A(t), \dot{A}(t))$ :

$$
\left(A, A^{\prime}\right) \mapsto \mathrm{D}_{2} \mathcal{L}\left(A, A^{\prime}\right) \Gamma(A) P^{\prime}=-\left\langle A^{\prime}, \mathrm{d}_{A} P^{\prime}\right\rangle .
$$

This provides an interpretation of the charge conservation property.

\section{Discretization}

\subsection{Unconstrained schemes}

- For the discussion of numerical schemes we suppose that the spaces $\Omega^{p}$ do not consist solely of smooth fields but have been completed with respect to some norm of the form:

$$
u \mapsto\|u\|_{\mathrm{L}^{q}}+\|\mathrm{d} u\|_{\mathrm{L}^{q}} .
$$

Then the exterior derivative extends to a continuous operator $\mathrm{d}: \Omega^{p} \rightarrow \Omega^{p+1}$. The exponent $q$ should be chosen such that the brackets of forms yield sufficiently integrable forms.

For each integer $p$ let $X_{h}^{p}$ denote a finite dimensional subspace of $\Omega^{p}$. We require that $\mathrm{d} X_{h}^{p} \subset X_{h}^{p+1}$. This provides a commuting diagram :

$$
\begin{aligned}
& 0 \rightarrow \Omega^{0} \rightarrow \Omega^{1} \rightarrow \Omega^{2} \rightarrow \cdots
\end{aligned}
$$

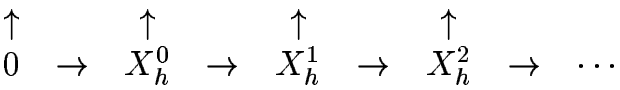

where the vertical arrows are inclusion maps and the horizontal ones are instances of the exterior derivative. The following results make most sense when 
also $X_{h}^{p+1}$ is small among the spaces containing $\mathrm{d} X_{h}^{p}$ (the precise statement would be that the vertical arrows in diagram (3.2) should induce isomorphisms in cohomology, see Gelfand-Manin [16]). Commuting diagrams are increasingly used to interpret or guide developments in numerical analysis, see Arnold [1].

The index $h$ is there to remind us that we are in fact interested in sequences of spaces indexed by some parameter $h$ and convergence results with respect to $h$.

For the numerical experiments we equip the domain $M$ with a simplicial mesh $\mathcal{T}_{h}$ and take $\left(X_{h}^{p}\right)$ to be the standard lowest order finite element complex, where $X_{h}^{0}$ consists of continuous and piecewise affine functions, $X_{h}^{1}$ is a space of 1 -forms called (lowest order) Nédélec's edge elements (of the first family) and $X_{h}^{2}$ is a space of 2 -forms referred to as Raviart-Thomas face elements. Similar constructions have been used in physics, see Sorkin [33] and can be traced back at least to Weil [38] who used them for algebraic/topological purposes (see also Whitney [39]). The interpretation of mixed finite element methods in terms of such differential forms is due to Bossavit [5]. In this choice of spaces the dimension of $X_{h}^{p}$ is exactly the number of $p$-dimensional simplexes in the mesh. The parameter $h$ denotes the maximal diameter of the simplexes of $\mathcal{T}_{h}$.

The finite element point of view provides other choices of spaces as well, for instance spaces built with polynomials of any degree, see Nédélec [28][29]. For surveys on these spaces in the context of electromagnetics, we refer to Hiptmair [19] and Monk [27]. So-called $h p$ versions of these finite element spaces are also useful for simulating Maxwell's equations and we refer to Demkowicz [11] for an overview on this topic. For generalities about mixed finite element methods we refer to [7][30][17].

Given such a finite dimensional complex we will use the spaces $X_{h}^{p} \otimes \mathfrak{g}$ as discretization spaces for Lie algebra valued differential forms.

- We consider time-dependent gauge potentials of the form $A: t \mapsto A(t) \in$ $X_{h}^{1} \otimes \mathfrak{g}$. Restricting the Lagrangian $(2.32)$ to $\left(X_{h}^{1} \otimes \mathfrak{g}\right)^{2}$, the corresponding stationary action principle yields the Euler-Lagrange equation:

$$
\forall A^{\prime} \in X_{h}^{1} \otimes \mathfrak{g} \quad\left\langle\ddot{A}, A^{\prime}\right\rangle=-\left\langle\mathcal{C}(A), \mathrm{d}_{A} A^{\prime}\right\rangle .
$$

An equivalent first order formulation is the following system for time-dependent $A, E$ in $X_{h}^{1} \otimes \mathfrak{g}:$

$$
\begin{aligned}
\dot{A} & =-E, \\
\forall E^{\prime} \in X_{h}^{1} \otimes \mathfrak{g} \quad\left\langle\dot{E}, E^{\prime}\right\rangle & =\left\langle\mathcal{C}(A), \mathrm{d}_{A} E^{\prime}\right\rangle .
\end{aligned}
$$

For these semi-discrete equations we have energy conservation. For instance for the second order formulation, along any trajectory $A$, the following quantity is constant:

$$
1 / 2\left(\|\dot{A}\|^{2}+\|\mathcal{C}(A)\|^{2}\right) .
$$

This can be checked directly by inserting $A^{\prime}=\dot{A}$ in (3.3) and is of course a general property of Hamiltonian systems.

We remark furthermore that we have the Bianchi identity:

$$
\mathrm{d}_{A} \mathcal{C}(A)=0,
$$

corresponding to the absence of magnetic charges, since $\mathcal{C}(A)$ is represented exactly (in both formulations). 
- The Bianchi identity corresponds to the constraint $\operatorname{div} H=0$ in electromagnetics. For the analogue of $\operatorname{div} E=0$ we have greater problems however. For discretizations of Maxwell's equations this constraint is obtained in a weak form. We now detail this, as a source of inspiration for Yang-Mills equations.

The standard semi-discretization of the Euler-Lagrange equation (2.50) for Maxwell's equations, would be to look for a magnetic potential of the form $A: t \mapsto A(t) \in X_{h}^{1}$ satisfying:

$$
\forall A^{\prime} \in X_{h}^{1} \quad\left\langle\ddot{A}, A^{\prime}\right\rangle=-\left\langle\operatorname{curl} A, \operatorname{curl} A^{\prime}\right\rangle .
$$

Inserting $A^{\prime}=\operatorname{grad} P^{\prime}$ for $P^{\prime} \in X_{h}^{0}$ into this equation, gives:

$$
\left\langle\dot{A}, \operatorname{grad} P^{\prime}\right\rangle^{\cdot}=0,
$$

when $P^{\prime}$ is considered as a constant function of time. In particular, if the equality $\left\langle\dot{A}, \operatorname{grad} P^{\prime}\right\rangle=0$ holds initially for some $P^{\prime} \in X_{h}^{0}$, it will hold at all times.

- If a magnetic potential $A \in X_{h}^{1}$ satisfies $\left\langle\dot{A}, \operatorname{grad} P^{\prime}\right\rangle=0$ for all $P^{\prime} \in X_{h}^{0}$, one says that it is weakly (or Galerkin) divergence free. For most purposes (practical or theoretical), weak divergence freedom for edge elements is just as good as strong divergence freedom. An important example is Kikuchi's compactness property [21]. It asserts that if we have a sequence $\left(A_{h}\right)$ indexed by a mesh parameter $h \rightarrow 0$, of fields $A_{h} \in X_{h}^{1}$ such that $A_{h}$ and curl $A_{h}$ are $\mathrm{L}^{2}$-bounded, and $A_{h}$ is weakly divergence free, one can extract a subsequence which is $\mathrm{L}^{2}$ convergent. This property mimics the (Rellich) compactness of the injection $\mathrm{H}^{1} \rightarrow \mathrm{L}^{2}$, since a vector-field on a compact manifold which is in $\mathrm{L}^{2}$ and has its curl and divergence in $\mathrm{L}^{2}$, is actually in $\mathrm{H}^{1}$. This property is an essential ingredient in the analysis of the numerical approximation of eigenvalues of the curl curl operator on edge element spaces, see Boffi et al. [3], and essentially guarantees the absence of spurious modes.

A refinement of Kikuchi's property which is useful for the convergence analysis of non-linear terms and which was partly inspired by the present work, is proved in Christiansen [9]. It indicates that with respect to the convergence of so called div-curl expressions, if $\left(A_{h}\right)$ is an $\mathrm{L}^{2}$-bounded sequence of vector fields $A_{h} \in X_{h}^{1}$ which are discrete divergence free, everything happens as if $\operatorname{div} A_{h} \rightarrow 0$ in the $\mathrm{H}^{-1}$ norm.

- For non-commutative Lie groups $G$ the simple trick used in (3.9) can no longer be carried out. One would like to be able to insert $A^{\prime}=\mathrm{d}_{A} P^{\prime}$ into (3.3), for $P^{\prime} \in X_{h}^{0} \otimes \mathfrak{g}$, but this is not legitimate since in general $\mathrm{d}_{A}$ maps $X_{h}^{0} \otimes \mathfrak{g}$ out of $X_{h}^{1} \otimes \mathfrak{g}$. From the point of view of Noether's theorem we remark that the gauge transformations associated with maps $Q: M \rightarrow G$ of the form:

$$
x \mapsto Q(x)=\exp (P(x)), \quad P \in X_{h}^{0} \otimes \mathfrak{g},
$$

do not map $X_{h}^{1} \otimes \mathfrak{g}$ into itself, contrary to the linear Maxwell case.

One might still hope that for any given $P^{\prime} \in X_{h}^{0} \otimes \mathfrak{g}$, considered as a constant function of time, the quantities $\left\langle\dot{A}, \mathrm{~d}_{A} P^{\prime}\right\rangle$ just oscillate around the initial value which is assumed to be small. We now present some numerical results to see if these hopes are answered. 
- We will show numerical results obtained with time discretizations according to the leap-frog scheme, which is by far the most popular time discretization of large scale Hamiltonian systems. For its use in the case of Maxwell's equations see Joly [20]. For additional points on the numerical analysis of time and space discretization of Maxwell's equations see Ciarlet-Zou [10]. This discretization scheme should be seen in the context of so-called symplectic integration methods, see Hairer et al. [18], Leimkuhler- Reich [25] and Marsden-West [26]. We introduce a time step $\tau$ and consider approximations $A^{i}$ of $A(i \tau)$ obtained by the recursion formula:

$$
\left\langle\frac{A^{i+1}-2 A^{i}+A^{i-1}}{\tau^{2}}, A^{\prime}\right\rangle=-\left\langle\mathcal{C}\left(A^{i}\right), \mathrm{DC}\left(A^{i}\right) A^{\prime}\right\rangle .
$$

Here and in the following all variables appearing with a "" should be preceded by a "for all" statement referring to a certain space of the form $X_{h}^{p} \otimes \mathfrak{g}$, which should be clear from the context. Hopefully this eases the notational burden somewhat.

The leap-frog scheme is usually implemented using coupled one-term recurrences rather than a two-term recurrence. One introduces $E^{i+1 / 2}$ as an approximation of $E((i+1 / 2) \tau)$, in the following reformulation of (3.11):

$$
\begin{aligned}
\frac{A^{i}-A^{i-1}}{\tau} & =-E^{i-1 / 2}, \\
\left\langle\frac{E^{i+1 / 2}-E^{i-1 / 2}}{\tau}, E^{\prime}\right\rangle & =\left\langle\mathcal{C}\left(A^{i}\right), \mathrm{DC}\left(A^{i}\right) E^{\prime}\right\rangle .
\end{aligned}
$$

Since $E$ and $A$ are not seen at the same time this technique is often called time staggering. To some extent time staggering is what lets us remain explicit in the time step, a huge advantage compared with implicit schemes for large scale computations.

- In Figure 1 we show numerical results obtained for $\mathfrak{s u}(2)$-valued gauge potentials on a 2-dimensional sphere. Thus in our previous notations $\mathfrak{g}=\mathfrak{s u}(2)$ and $M=\mathbb{S}^{2}$. Recall that $\mathfrak{s u}(2)$ is the three-dimensional Lie algebra consisting of trace-free and skew-hermitian $2 \times 2$ complex matrices (see e.g. Duisterman-Kolk [13] p. 10). It is the Lie algebra associated with the compact Lie group SU(2) consisting of unitary $2 \times 2$ complex matrices with determinant 1 .

We have plotted the $\mathrm{L}^{2}$ norms squared of $A^{i}, \mathrm{~d} A^{i}$ and $(1 / 2)\left[A_{i}, A_{i}\right]$ as a function of time in multiples of $\pi$. For the chosen initial values the $\mathrm{L}^{2}$-norm of the quadratic term $(1 / 2)\left[A_{i}, A_{i}\right]$ is relatively small compared with the linear term $\mathrm{d} A^{i}$ in the curvature. Linearization of the equations indicates that the waves travel around the sphere with speed 1 , which accounts some of the almost $2 \pi$-periodic features which can be seen in the graphs.

For Maxwell's equations (3.8) the leap-frog discretization is stable under a CFL condition (i.e. $\tau \leq C h$ ). This is proved in Joly [20] using the conservation of a discrete energy containing a negative term which is controlled by an inverse inequality. This discrete energy is defined as:

$$
(1 / 2)\left\|\frac{A^{i+1}-A^{i}}{\tau}\right\|^{2}+(1 / 2)\left(\left\|\operatorname{curl}\left(\frac{A^{i+1}+A^{i}}{2}\right)\right\|^{2}-\left\|\operatorname{curl}\left(\frac{A^{i+1}-A^{i}}{2}\right)\right\|^{2}\right) .
$$

For the general Yang-Mills equations we have not found a similar conserved discrete energy. However at least for the numerical examples, the leap-frog 


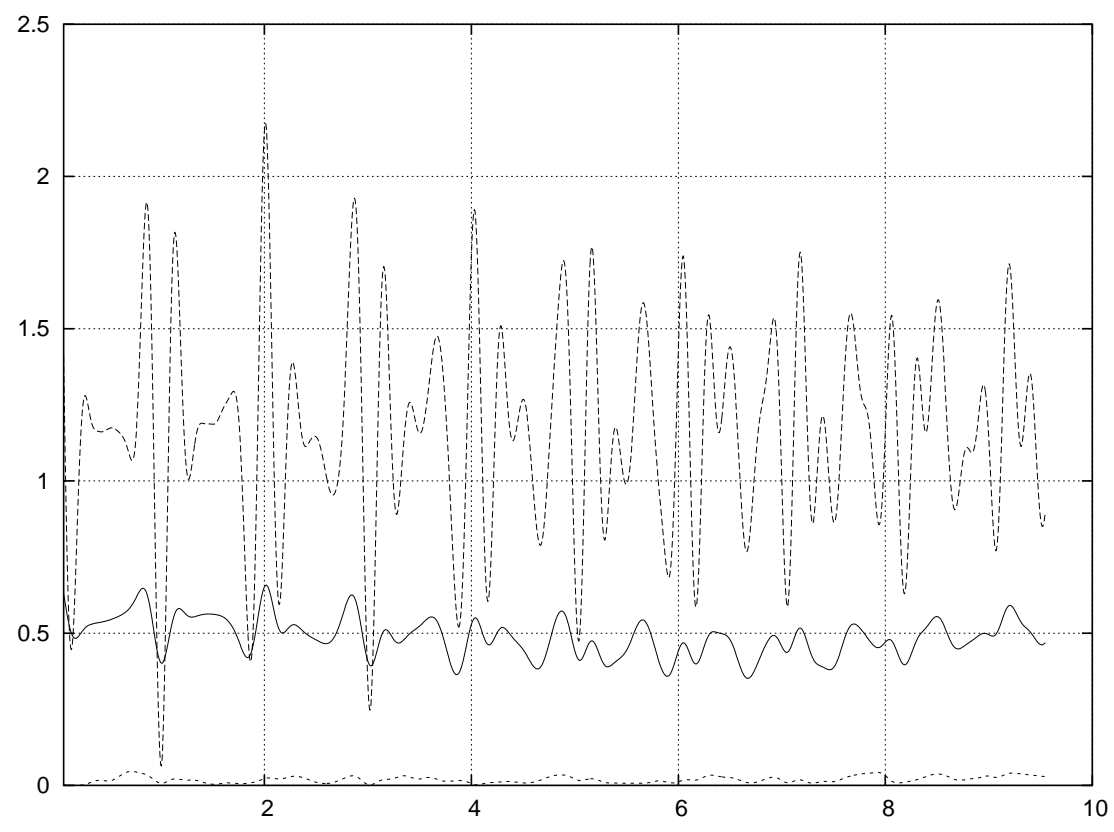

Figure 1: $\mathrm{L}^{2}$ norms squared of $A^{i}$ (plain), $\mathrm{d} A^{i}$ (dashed) and $(1 / 2)\left[A_{i}, A_{i}\right]$ (finely dashed).

scheme seems to be stable for roughly the same values of the time step $\tau$ as for the Maxwell case. For the numerical results presented here the time step is 0.01 , which is a few times less than the stability limit. The graphs show 3000 time-steps.

In Figure 2 we have plotted some reasonable approximations of the energy as a function of time. These are:

$$
\mathcal{E}^{i+1 / 2}=(1 / 2)\left\|\frac{A^{i+1}-A^{i}}{\tau}\right\|^{2}+(1 / 4)\left(\left\|\mathcal{C}\left(A^{i}\right)\right\|^{2}+\left\|\mathcal{C}\left(A^{i+1}\right)\right\|^{2}\right),
$$

and:

$$
\mathcal{E}^{i}=(1 / 2)\left\|\frac{A^{i+1}-A^{i-1}}{2 \tau}\right\|^{2}+(1 / 2)\left\|\mathcal{C}\left(A^{i}\right)\right\|^{2} .
$$

We have also plotted a linear combination of these two energies which yielded very good conservation (the conservation cannot be exact however for convex combinations, since this would imply unconditional stability).

In Figure 3 we have plotted two graphs. One is the $\mathrm{L}^{2}$-norm squared of a discrete divergence field $D^{i} \in X_{h}^{0} \otimes \mathfrak{g}$ defined as the solution of (for all $D^{\prime} \in$ $\left.X_{h}^{0} \otimes \mathfrak{g}\right):$

$$
\left\langle D^{i}, D^{\prime}\right\rangle=\left\langle\frac{A^{i+1}-A^{i-1}}{2 \tau}, \mathrm{d} D^{\prime}\right\rangle .
$$

The other graph is the $\mathrm{L}^{2}$-norm squared of a field $C^{i} \in X_{h}^{0} \otimes \mathfrak{g}$ measuring charge defined by (for all $C^{\prime} \in X_{h}^{0} \otimes \mathfrak{g}$ ):

$$
\left\langle C^{i}, C^{\prime}\right\rangle=\left\langle\frac{A^{i+1}-A^{i-1}}{2 \tau}, \mathrm{d}_{A^{i}} C^{\prime}\right\rangle .
$$




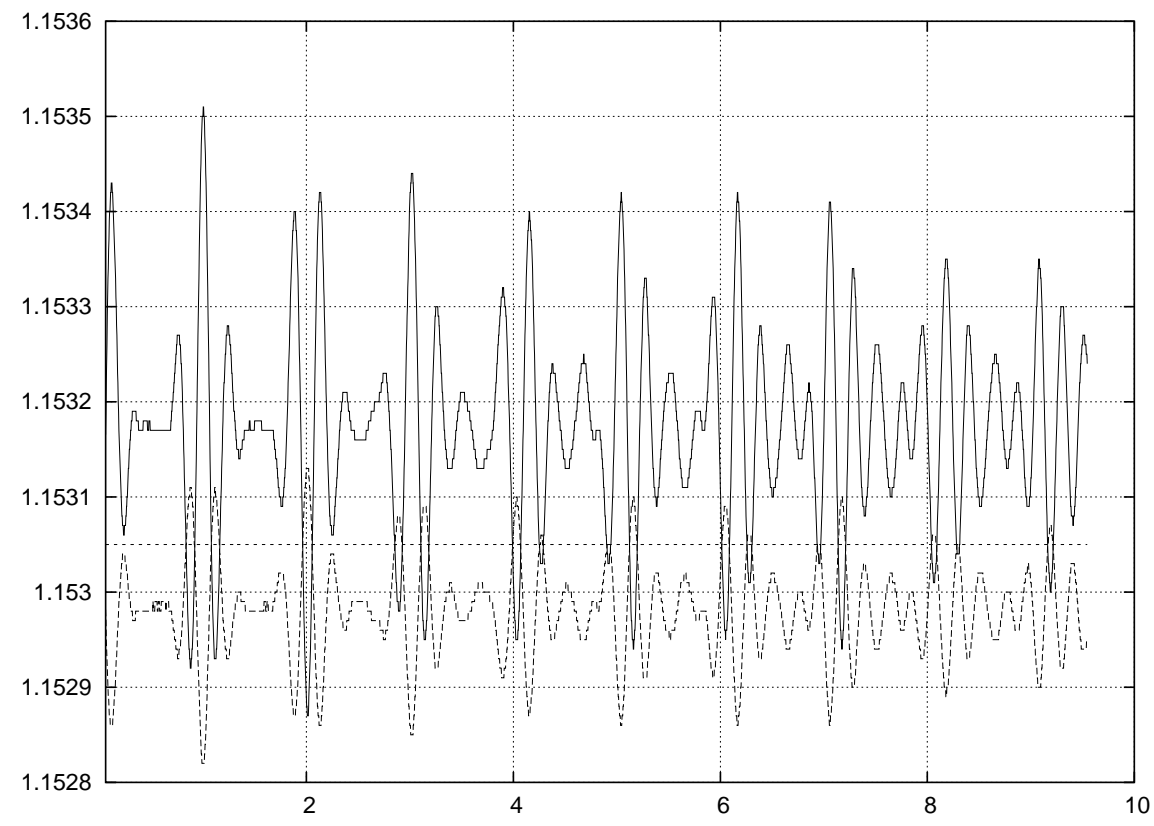

Figure 2: Energies $\mathcal{E}^{i-1 / 2}$ (plain), $\mathcal{E}^{i}$ (dashed) and $(1 / 6)\left(\mathcal{E}^{i-1 / 2}+4 \mathcal{E}^{i}+\mathcal{E}^{i+1 / 2}\right)$ (finely dashed).

Ideally the charge field $C^{i}$ should remain small compared with $D^{i}$, but this appears not to be the case. This tends to indicate that also the field $D^{i}$ is unreliable and direct visualization shows that it displays strongly mesh-dependent features.

We believe this bad behavior to be attributable to the semi-discretization (the space discretization) rather than the leap-frog scheme (the time discretization). That is, the leap frog scheme just serves to illustrate that the semidiscretzation does not provide adequate charge conservation. In this sense the semi-discretization (3.3) is inadequate for simulating Yang-Mills equations. Note that the stability indicated by Figures 1 and 2, together with the drift observed in Figure 3, illustrates the fact that the Yang-Mills system is stable also away from the constraint manifold.

- In this paper we will mostly use the leap-frog scheme. This is of course not the only possibility. As an example of an interesting implicit scheme we mention:

$$
\begin{gathered}
\left\langle\frac{A^{i+1}-2 A^{i}+A^{i-1}}{\tau^{2}}, A^{\prime}\right\rangle= \\
-\frac{1}{2}\left\langle\mathcal{C}\left(\frac{A^{i-1}+A^{i}}{2}\right)+\mathcal{C}\left(\frac{A^{i}+A^{i+1}}{2}\right), \mathrm{DC}\left(\frac{A^{i-1}}{4}+\frac{A^{i}}{2}+\frac{A^{i+1}}{4}\right) A^{\prime}\right\rangle .
\end{gathered}
$$

One of its charms is the following discrete energy conservation property: 


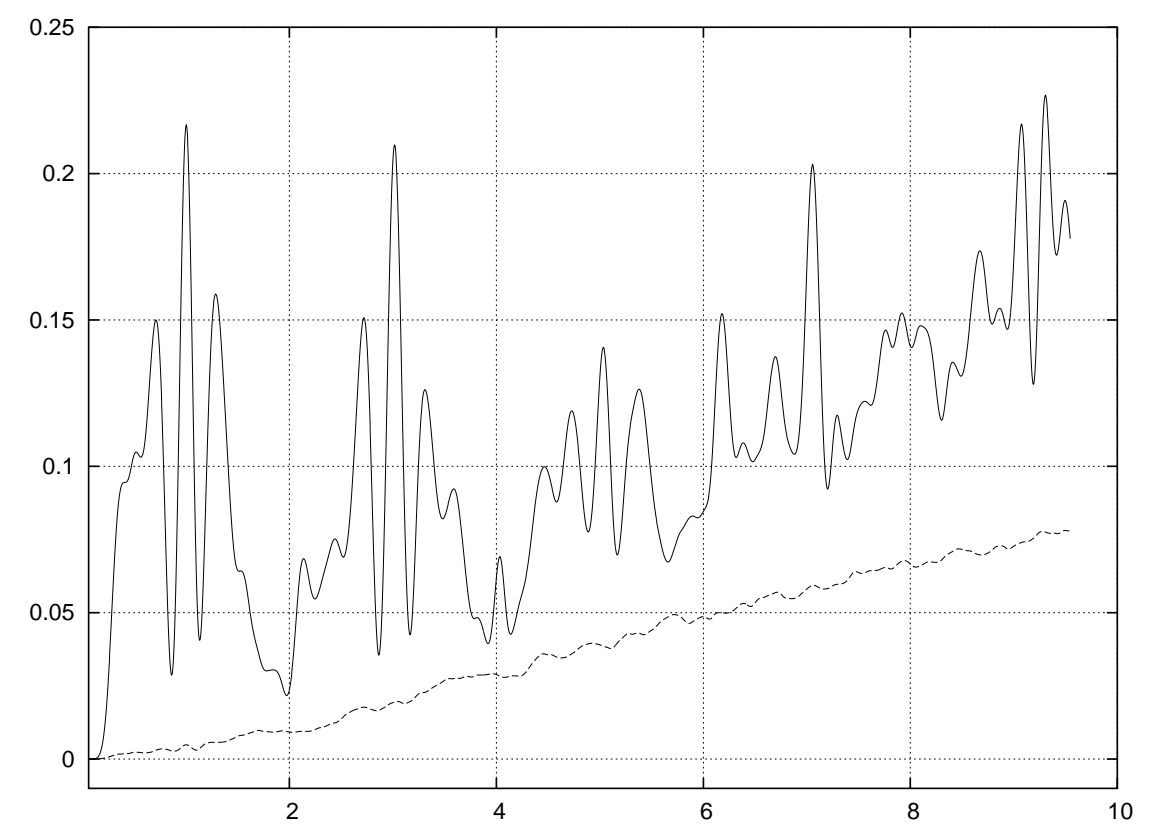

Figure 3: $\mathrm{L}^{2}$ norms squared of divergence $D^{i}$ (plain) and charge $C^{i}$ (dashed).

Proposition 3.1 The implicit scheme (3.19, 3.20) has the following conserved energy:

$$
\frac{1}{2}\left(\left\|\frac{A^{i+1}-A^{i}}{\tau}\right\|^{2}+\left\|\mathcal{C}\left(\frac{A^{i+1}+A^{i}}{2}\right)\right\|^{2}\right) .
$$

- Proof: Inserting $A^{\prime}$ defined by:

$$
A^{\prime}=\frac{A^{i+1}-A^{i-1}}{2 \tau}=\frac{1}{2}\left(\frac{A^{i+1}-A^{i}}{\tau}+\frac{A^{i}-A^{i-1}}{\tau}\right),
$$

into the left hand side (3.19) gives:

$$
\frac{1}{2 \tau}\left(\left\|\frac{A^{i+1}-A^{i}}{\tau}\right\|^{2}-\left\|\frac{A^{i}-A^{i-1}}{\tau}\right\|^{2}\right) .
$$

Next we use the property that since the curvature map $\mathcal{C}$ is a polynomial of degree 2 we have, for all gauge potentials $A, B$ :

$$
\mathcal{C}(B)=\mathcal{C}(A)+\mathrm{DC}\left(\frac{A+B}{2}\right)(B-A) .
$$

Applying this identity with:

$$
A=\frac{A^{i-1}+A^{i}}{2} \text { and } B=\frac{A^{i}+A^{i+1}}{2}
$$

shows that the right hand side (3.20) with $A^{\prime}=(1 / \tau)(B-A)$ is equal to :

$$
-\frac{1}{2 \tau}\left\langle\mathcal{C}\left(\frac{A^{i-1}+A^{i}}{2}\right)+\mathcal{C}\left(\frac{A^{i}+A^{i+1}}{2}\right), \mathcal{C}\left(\frac{A^{i}+A^{i+1}}{2}\right)-\mathcal{C}\left(\frac{A^{i-1}+A^{i}}{2}\right)\right\rangle
$$


From this the proposition follows.

This conservation property implies unconditional stability of the scheme. This is useful in particular when the mesh contains strong local refinements.

\subsection{Constrained schemes}

- Since unconstrained schemes have trouble with charge conservation we now examine ways to impose this as a constraint on the flow. This is easier to do on first order formulations such as $(3.4,3.5)$.

In analogy with the continuous case and standard discretizations of Maxwell's equations, we would like $(A, E)$ to evolve on the subset of $\left(X_{h}^{1} \otimes \mathfrak{g}\right) \times\left(X_{h}^{1} \otimes \mathfrak{g}\right)$ with equation:

$$
\forall P^{\prime} \in X_{h}^{0} \otimes \mathfrak{g} \quad\left\langle E, \mathrm{~d}_{A} P^{\prime}\right\rangle=0 .
$$

One expects this set to be manifold-like, with singularities located where the kernel of $\mathrm{d}_{A}$ is non-trivial.

- We first discuss constraint imposition in general. Suppose we have a system of the form:

$$
\dot{u}=f(u), \quad g(u)=0,
$$

which is compatible in the sense that the vectorfield $f$ is tangent to the zeroset $Z_{g}$ of $g$. One way of imposing constraints on this system is by introducing Lagrange multipliers $v$ and impose that for all $u^{\prime}, v^{\prime}$ :

$$
\begin{aligned}
\left\langle\dot{u}, u^{\prime}\right\rangle+\left\langle\mathrm{D} g(u) u^{\prime}, v\right\rangle & =\left\langle f(u), u^{\prime}\right\rangle, \\
\left\langle\mathrm{D} g(u) \dot{u}, v^{\prime}\right\rangle & =0 .
\end{aligned}
$$

When $\mathrm{D} g$ is onto at each point the system has a unique solution, for given initial values. If the constraint is satisfied initially, it produces trajectories on the zeroset $Z_{g}$ of $g$, governed by the vector field defined as the orthogonal projection of $f$ to the tangent spaces of $Z_{g}$, according to the duality products $\langle\cdot, \cdot\rangle$. More generally it produces trajectories on the level-sets of $g$.

When the constraint is compatible, this trajectory must be the one given by (3.28) and the Lagrange multiplier $v$ remains equal to 0 . But when the system $(3.29,3.30)$ is used for incompatible systems, the Lagrange multiplier $v$ will vary, measuring in a sense how incompatible the original system was.

- If we apply this method of imposing constraints, to the first order system $(3.4,3.5)$ with the constraint $(3.27)$, we get the following constrained first order system, with Lagrange multipliers $P: t \mapsto P(t) \in X_{h}^{0} \otimes \mathfrak{g}$ :

$$
\begin{aligned}
\left\langle\dot{A}, A^{\prime}\right\rangle+\left\langle E,\left[A^{\prime}, P\right]\right\rangle & =\left\langle-E, A^{\prime}\right\rangle, \\
\left\langle\dot{E}, E^{\prime}\right\rangle+\left\langle E^{\prime}, \mathrm{d}_{A} P\right\rangle & =\left\langle\mathcal{C}(A), \mathrm{d}_{A} E^{\prime}\right\rangle, \\
\left\langle E,\left[\dot{A}, P^{\prime}\right]\right\rangle+\left\langle\dot{E}, \mathrm{~d}_{A} P^{\prime}\right\rangle & =0 .
\end{aligned}
$$

In this system (3.31) can be rewritten using (2.31):

$$
\dot{A}=-E+\Pi_{h}[E, P],
$$

where $\Pi_{h}$ denotes $\mathrm{L}^{2}$-orthogonal projection onto $X_{h}^{1} \otimes \mathfrak{g}$. In this situation it is not clear what one should call energy of the system, let alone if it is conserved. 
This is a severe drawback. One could impose an additional constraint on the energy, but this seems cumbersome. Moreover explicit time discretization of the constrained system $(3.31-3.33)$ seems difficult to achieve since the constraint (3.33) appears to be unsuited for time-staggering as in $(3.12,3.13)$.

- In the system $(3.31-3.33)$ we see that the term $\left\langle E,\left[\dot{A}, P^{\prime}\right]\right\rangle$ ought to be 0 . As an alternative to that system we therefore consider the following non-standard constrained system for fields $A(t), E(t) \in X_{h}^{1} \otimes \mathfrak{g}$ and Lagrange multipliers $P(t) \in X_{h}^{0} \otimes \mathfrak{g}:$

$$
\begin{aligned}
\dot{A} & =-E, \\
\left\langle\dot{E}, E^{\prime}\right\rangle+\left\langle E^{\prime}, \mathrm{d}_{A} P\right\rangle & =\left\langle\mathcal{C}(A), \mathrm{d}_{A} E^{\prime}\right\rangle, \\
\left\langle\dot{E}, \mathrm{~d}_{A} P^{\prime}\right\rangle & =0 .
\end{aligned}
$$

For the continuous analogue of this system, we remark that by the cancellation property (2.54) given in our first proof of charge conservation in $\S 2.3$, it is equivalent to the unconstrained Yang-Mills equations (2.35) and should yield Lagrange multipliers equal to 0.

For the discrete case we obtain a system which is both energy and constraint preserving (in particular it is not equivalent to the unconstrained semidiscretization):

Proposition 3.2 For any solution of (3.35 - 3.37), if the constraint (3.27) is satisfied initially, it will be satisfied at all times, and we have energy conservation:

$$
1 / 2\left(\|E\|^{2}+\|\mathcal{C}(A)\|^{2}\right)^{\cdot}=0 .
$$

- Proof: Indeed we have for constant $P^{\prime}$ :

$$
\left\langle E, \mathrm{~d}_{A} P^{\prime}\right\rangle^{\cdot}=\left\langle\dot{E}, \mathrm{~d}_{A} P^{\prime}\right\rangle+\left\langle E,\left[E, P^{\prime}\right]\right\rangle=0 .
$$

It follows that if the constraint (3.27) is satisfied initially it will be satisfied at all times. Inserting $E^{\prime}=E$ in (3.36) then gives energy conservation.

- Next we turn to time discretizations of $(3.35-3.37)$. Consider the following scheme:

$$
\begin{aligned}
\frac{A^{i}-A^{i-1}}{\tau} & =-E^{i-1 / 2}, \\
\left\langle\frac{E^{i+1 / 2}-E^{i-1 / 2}}{\tau}, E^{\prime}\right\rangle+\left\langle E^{\prime}, \mathrm{d}_{A^{i}} P^{i}\right\rangle & =\left\langle\mathcal{C}\left(A^{i}\right), \mathrm{DC}\left(A^{i}\right) E^{\prime}\right\rangle, \\
\left\langle\frac{E^{i+1 / 2}-E^{i-1 / 2}}{\tau}, \mathrm{d}_{A^{i}} P^{\prime}\right\rangle & =0 .
\end{aligned}
$$

Luckily this scheme turns out to be constraint preserving in the following sense:

Proposition 3.3 For any solution of $(3.40-3.42)$ the following quantities are preserved:

$$
\left\langle\frac{A^{i+1}-A^{i-1}}{2 \tau}, \mathrm{d}_{A^{i}} P^{\prime}\right\rangle .
$$


- Proof: First we remark that:

$$
\begin{aligned}
\left\langle\frac{A^{i+1}-A^{i-1}}{2 \tau}, \mathrm{d}_{A^{i}} P^{\prime}\right\rangle & =\left\langle\frac{E^{i+1 / 2}+E^{i-1 / 2}}{2}, \mathrm{~d}_{A^{i}} P^{\prime}\right\rangle \\
& =\left\langle E^{i+1 / 2}, \mathrm{~d}_{A^{i}} P^{\prime}\right\rangle=\left\langle E^{i-1 / 2}, \mathrm{~d}_{A^{i}} P^{\prime}\right\rangle
\end{aligned}
$$

Then we write:

$$
\begin{aligned}
\left\langle E^{i+1 / 2}, \mathrm{~d}_{A^{i}} P^{\prime}\right\rangle-\left\langle E^{i-1 / 2}, \mathrm{~d}_{A^{i-1}} P^{\prime}\right\rangle & =\left\langle E^{i-1 / 2},\left[A^{i}-A^{i-1}, P^{\prime}\right]\right\rangle, \\
& =\left\langle E^{i-1 / 2},\left[-\tau E^{i-1 / 2}, P^{\prime}\right]\right\rangle \\
& =0
\end{aligned}
$$

This gives the desired result.

Next we show numerical resuts for the same mesh, time step and initial values as for the unconstrained case. In Figure 4 we have plotted $\mathrm{L}^{2}$ norms as in Figure 1 and obtained graphs which are very similar but not identical to the unconstrained case.

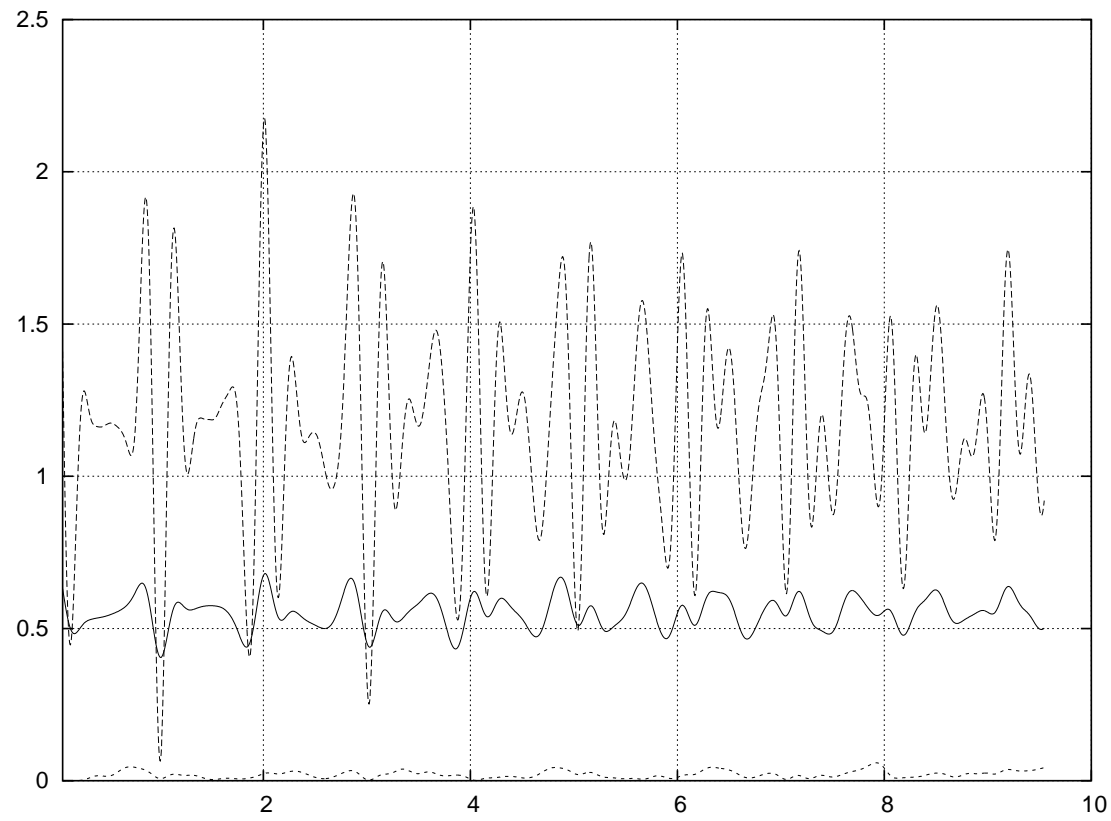

Figure 4: $\mathrm{L}^{2}$ norms squared of $A^{i}$ (plain), $\mathrm{d} A^{i}$ (dashed) and (1/2)[ $\left.A_{i}, A_{i}\right]$ (finely dashed). Constrained case.

In Figure 5 we have plotted the same energies as in Figure 2. Here the similarity with the unconstrained case is surprising. Finally in Figure 6 we have plotted the divergence and charge defined as for Figure 3. It appears that we have obtained charge conservation without destroying long-time energy conservation. This is somewhat surprising since for symplectic schemes such as the leap frog scheme existing results on long-time approximate energy conservation are often based on fine properties of Hamiltonian ODEs (see in particular [18], 
chapter 13) whose extension to constrained discretizations of a PDE are not clear. For the plot of the divergence we have rather large discrepancies with the unconstrained case.

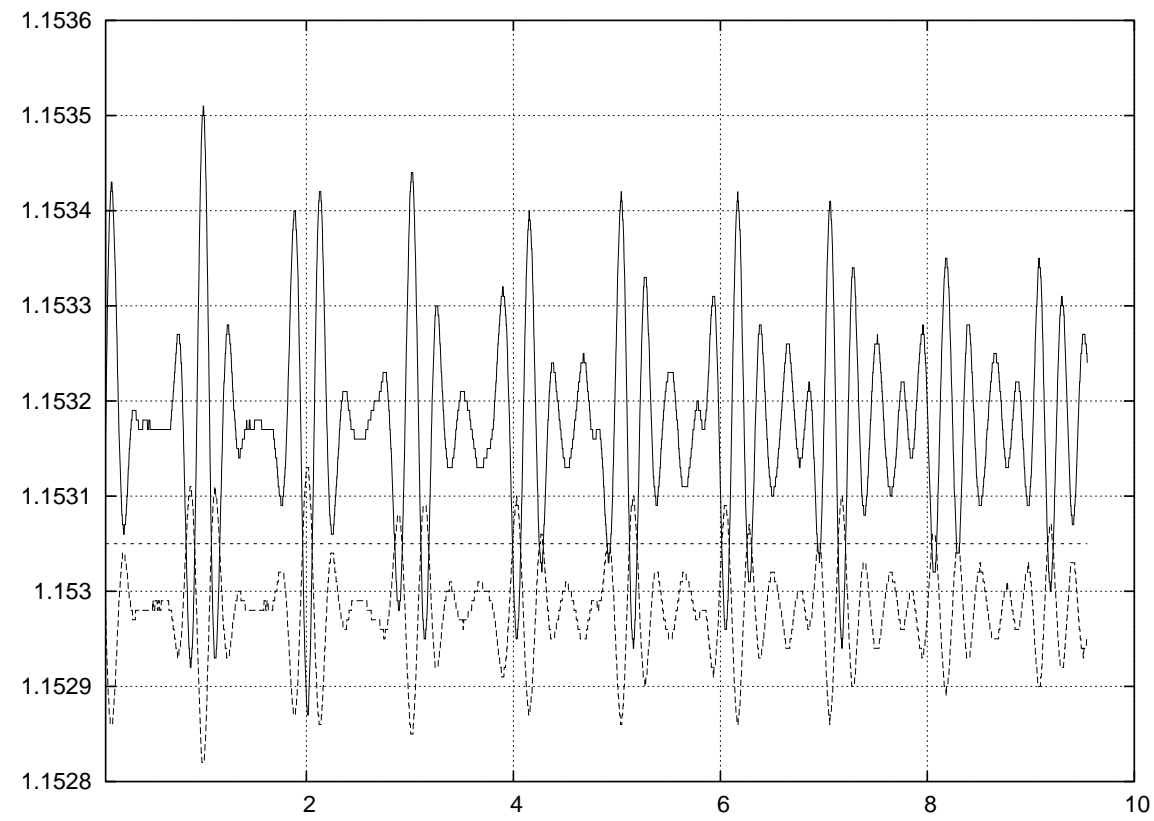

Figure 5: Energies $\mathcal{E}^{i+1 / 2}$ (plain), $\mathcal{E}^{i}$ (dashed) and $(1 / 6)\left(\mathcal{E}^{i-1 / 2}+4 \mathcal{E}^{i}+\mathcal{E}^{i+1 / 2}\right)$ (finely dashed). Constrained case.

- The above constraint preservation is not limited to the leap-frog scheme. The system $(3.40-3.42)$ can be expressed as a second order recurrence relation. The discrete acceleration:

$$
B^{i}=\frac{A^{i+1}-2 A^{i}+A^{i-1}}{\tau^{2}}
$$

should be a solution of:

$$
\begin{aligned}
\left\langle B^{i}, A^{\prime}\right\rangle+\left\langle A^{\prime}, \mathrm{d}_{A^{i}} P^{i}\right\rangle & =-\left\langle\mathcal{C}\left(A^{i}\right), \mathrm{DC}\left(A^{i}\right) A^{\prime}\right\rangle, \\
\left\langle B^{i}, \mathrm{~d}_{A^{i}} P^{\prime}\right\rangle & =0 .
\end{aligned}
$$

The proof of constraint preservation provided for Proposition 3.3 did not depend on the structure of the first term on the righthand side of (3.40). Therefore we can also impose constraints on the implicit scheme (3.19-3.20) by replacing the first term in the right hand side of eq. (3.50) by the one in (3.20). For this scheme, if the constraint is satified initially it will be satified for all times, enabling us keep the discrete energy conservation property proved in Proposition 3.1 . 


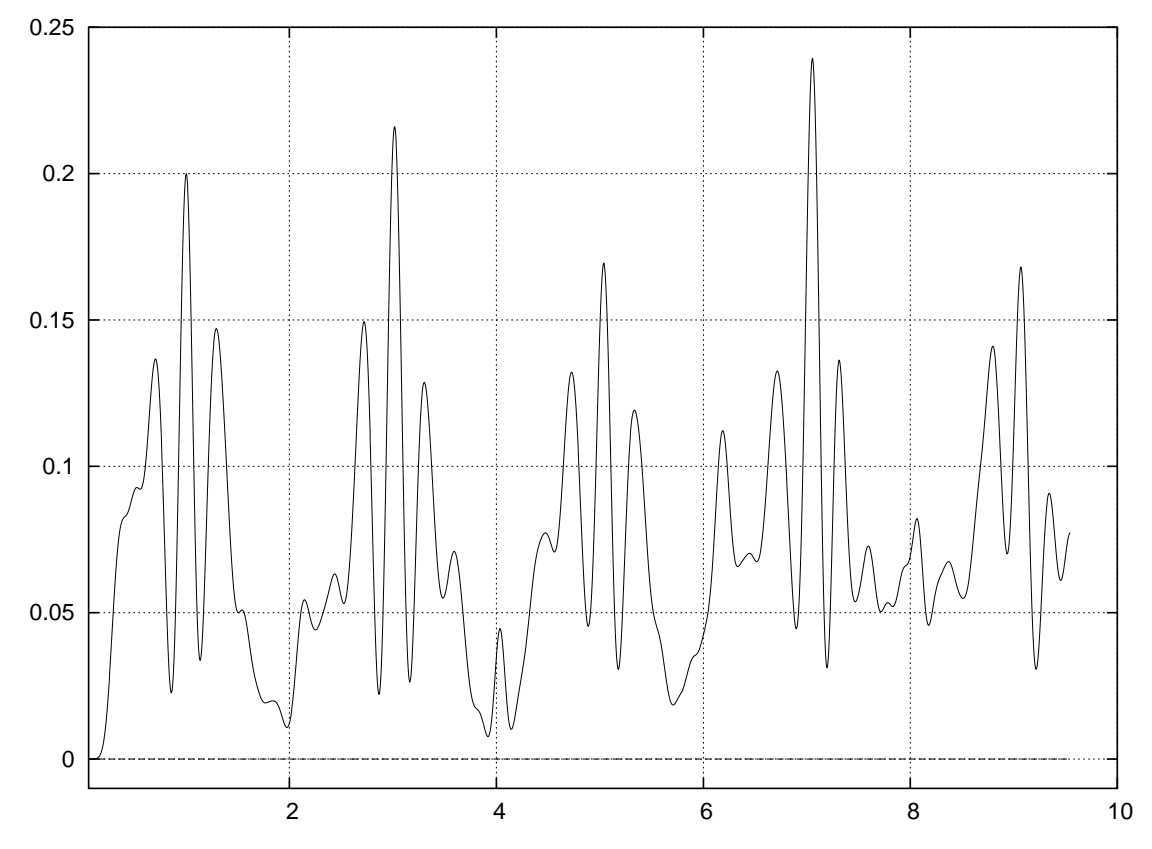

Figure 6: $\mathrm{L}^{2}$ norms squared of divergence $D^{i}$ (plain) and charge $C^{i}$ (dashed). Constrained case.

\subsection{Implementation questions}

- In this section we comment on some points relevant to the implementation of the above fully discrete algorithm: representation of bilinear maps and resolution of saddlepoint problems.

Several bilinear maps appear. In particular we have the wedge of differential forms, the Lie bracket on the chosen Lie algebra, and the bracket of Lie algebra valued differential forms, which is the tensor product of the preceding two maps. Concerning the Lie algebra, a basis $\left(e_{i}\right)$ is chosen and the bilinear map $[\cdot, \cdot]$ is expressed in this basis by the so-called structure constants $\beta_{i j}^{k}$ defined by:

$$
\left[e_{i}, e_{j}\right]=\sum_{k} \beta_{i j}^{k} e_{k} .
$$

A similar method can be used to represent the wedge of forms. We are interested in the case of the wedge on $X_{h}^{0} \times X_{h}^{1}$ and $X_{h}^{1} \times X_{h}^{1}$. From the first one, we can obtain the bracket of Lie valued forms needed to define the constraint by taking the Kronecker product with the structure constants of the Lie algebra. From the second we obtain the bracket needed to define curvature.

Products of polynomials create polynomials of higher degree so in order to represent these wedge products we must introduce high-order finite-element spaces. For simplicity we now skip the index $h$ for the spaces, and intoduce a new one referring to polynomial degree. For the lowest order finite element spaces already introduced we use $X^{p}=X_{1}^{p}$.

Denote by $X_{n}^{0}$ the space of continuous functions which are piecewise polynomials of degree at most $n$. We can also denote by $X_{0}^{0}$ the vector space consisting 
of the constant functions. The space $X_{n}^{0}$ can also be characterized as the vectorspace generated by products $u_{1} \cdots u_{n}$ where $u_{i} \in X_{1}^{0}=X^{0}$. Next we define the high order finite element space $X_{n+1}^{p}$ as the vector space generated by forms $u v$ where $u \in X_{n}^{0}$ and $v \in X_{1}^{p}$. Then one checks that:

$$
\wedge: X_{n}^{p} \times X_{m}^{q} \rightarrow X_{n+m}^{p+q} .
$$

It can also be checked that the spaces $X_{n}^{p}$ correspond to the first family of mixed finite element spaces as defined in [28], $n$ being the maximum degree of the polynomials used (in this context one often says that one uses incomplete polynomials of degree $n$ since not all differential forms which can be represented piecewise by polynomials of degree $n$ are used, witness the case $n=1$ ).

As already mentioned, to implement our algorithm we are interested in the cases:

$$
\wedge: X_{1}^{0} \times X_{1}^{1} \rightarrow X_{2}^{1} \text { and } \wedge: X_{1}^{1} \times X_{1}^{1} \rightarrow X_{2}^{2} .
$$

Implementing a basis for high-order finite elements is notoriously cumbersome, since it involves non-canonical choices, which would make for acrobatic indexing exercises when implementing the above wedge products. As a simpler alternative, instead of basises we used generating families which might have linear dependencies. Generally speaking, given a bilinear map $\alpha: E \times F \rightarrow G$ between finite dimensional spaces $E, F$ and $G$ equipped with finite generating families $\left(e_{i}\right),\left(f_{j}\right)$ and $\left(g_{k}\right)$ respectively, suppose we have some family $\left(\alpha_{i j}^{k}\right)$ such that:

$$
\alpha\left(e_{i}, f_{j}\right)=\sum_{k} \alpha_{i j}^{k} g_{k}
$$

Such a family exists but is in general not unique, but the action of $\alpha$ on vectors expressed as linear combinations of the families $\left(e_{i}\right),\left(f_{j}\right)$ can still be recovered. Explicitely, if:

$$
u=\sum_{i} u^{i} e_{i}, \quad \text { and } \quad v=\sum_{j} v^{j} f_{j},
$$

then we have, by simple bilinearity:

$$
\alpha(u, v)=w=\sum_{k} w^{k} g_{k}, \quad \text { with } \quad w_{k}=\sum_{i j} \alpha_{i j}^{k} u^{i} v^{j} .
$$

We now detail the choice of generating families for $\wedge: X_{1}^{0} \times X_{1}^{1} \rightarrow X_{2}^{1}$. For $E=X_{1}^{0}$ and $F=X_{1}^{1}$ we equip them with their standard (canonical) basises denoted $\left(e_{i}\right)_{i \in I}$ and $\left(f_{j}\right)_{j \in J}$ indexed by nodes and edges respectively. A generating family of $X_{2}^{1}$ is provided by the family $\left(e_{i} f_{j}\right)_{(i, j) \in I \times J}$ but most of these elements are 0 . We therefore use the subfamily consisting of: on the one hand, for each edge $j \in J$ with extremities $j_{0}$ and $j_{1}$ (members of $I$ ) in some orientation of the edges, the two 1-forms:

$$
e_{j_{0}} f_{j}, \quad \text { and } e_{j_{1}} f_{j}
$$

and on the other hand, for each triangle $k$ with vertices $k_{0}, k_{1}$ and $k_{2}$, if we denote by $k_{l}^{\prime}$ the edge opposite to the vertex $k_{l}$ :

$$
e_{k_{l}} f_{k_{l}^{\prime}} \quad \text { with } \quad l=0,1,2 .
$$


This last family is not linearly independent since for each triangle:

$$
\sum_{l} e_{k_{l}} f_{k_{l}^{\prime}}=0
$$

As already indicated, it turned out that it was easier to implement the algorithms in this framework rather than ejecting one of the three terms $e_{k_{l}} f_{k_{l}^{\prime}}$ in each triangle (which is enough to ensure linear independence).

- Working with tensors of the form $\left(\alpha_{i j}^{k}\right)$ requires routines for treating sparse tensors. For each $k$ there is only a small number (independent of $h$ ) of non-zero coefficients. For each $k$ we introduce sets $I_{k}$ and $J_{k}$ defined by:

$$
\begin{aligned}
& I_{k}=\left\{i \in I: \exists j \in J \quad \alpha_{i j}^{k} \neq 0\right\}, \\
& J_{k}=\left\{j \in J: \exists i \in I \quad \alpha_{i j}^{k} \neq 0\right\} .
\end{aligned}
$$

We number the elements of $I_{k}$ and $J_{k}$ consecutively with integers starting at 1 , and store for each element of the $\left|I_{k}\right| \times\left|J_{k}\right|$ matrix thus obtained, the corresponding coefficient $\alpha_{i j}^{k}$.

While this is certainly not the sparsest representation of the family $\left(\alpha_{i j}^{k}\right)$ it makes the following contraction operation rather quick and easy. In order to assemble the constraint matrix appearing in the saddlepoint problem (3.41, 3.42) we need to assemble the sparse matrix obtained by contracting the bracket ${ }^{1}$ $[\cdot, \cdot]:\left(X_{1}^{0} \otimes \mathfrak{g}\right) \times\left(X_{1}^{1} \otimes \mathfrak{g}\right) \rightarrow X_{2}^{1} \otimes \mathfrak{g}$ with a gauge potential $A^{i} \in X_{1}^{1} \otimes \mathfrak{g}$. In the above notations, if $\alpha$ is the bracket and $v$ is the gauge potential we want to assemble the matrix of the operator $u \mapsto \alpha(u, v)$. The sparsity pattern of this matrix is determined by the sets $I_{k}$ and assembling this matrix involves only summing the products:

$$
\sum_{j \in J_{k}} \alpha_{i j}^{k} v^{j}
$$

for each $k$ and each $i \in I_{k}$. This is a small number (independent of $h$ ) of operations for each $k$.

- In order to treat the saddlepoint problem $(3.41,3.42)$ we need not only assemble the matrix but of course also solve the resulting system. To do this we used an Usawa algorithm. It is an iterative algorithm which requires preconditioning the Schur complement. Indeed computing the Lagrange multiplier $P^{i}$ is essentially equivalent to solving a problem of the form:

$$
\forall P^{\prime} \in X_{h}^{0} \otimes \mathfrak{g} \quad\left\langle\Pi_{h} \mathrm{~d}_{A^{i}} P^{\prime}, \mathrm{d}_{A^{i}} P^{i}\right\rangle=L^{i}\left(P^{\prime}\right) .
$$

where $\Pi_{h}$ denotes $L^{2}$ projection $X_{2}^{1} \otimes \mathfrak{g} \rightarrow X_{1}^{1} \otimes \mathfrak{g}$, and $L^{i}$ is some linear form on $X_{1}^{0} \otimes \mathfrak{g}$ (this can be checked by directly inserting $E^{\prime}=\Pi_{h} \mathrm{~d}_{A^{i}} P^{\prime}$ into the system). For the numerical results presented here we used as a preconditioner the exact inverse of the matrix of the bilinear form on $\left(X_{1}^{0} \otimes \mathfrak{g}\right)^{2}$ defined as:

$$
\left(P, P^{\prime}\right) \rightarrow\left\langle\mathrm{d} P, \mathrm{~d} P^{\prime}\right\rangle+\left\langle P, P^{\prime}\right\rangle .
$$

\footnotetext{
${ }^{1}$ The $\mathrm{L}^{2}$ duality should also be incorporated in the discussion through its Gram (or mass) matrix on $X_{2}^{1} \otimes \mathfrak{g}$ but this is secondary for the point we want to make here.
} 
This inverse can be represented by factorization once the nodes have been optimally numbered. The number of times this matrix is used justifies the use of a factorization algorithm, as compared with iterative methods which often preferred for large scale problems. The above choice of preconditioner was guided by the fact that lower order terms are compact pertubrations of the Laplacian, so that one expects spectral clustering around 1 for the preconditioned system. The Babuska-Brezzi compatibility condition we will prove in the next section also proves that this preconditioner yields a condition number bounded uniformly in $h$.

- We now prove a Babuska-Brezzi compatibility condition, ensuring that the above saddlepoint problem $(3.41,3.42)$ (or equivalently $(3.64)$ ) is well-posed. It is slightly more technical than usual since $\mathrm{d}_{A}$ might have a non-trivial kernel. Recall that a continuous operator is said to be left semi-Fredholm if it has closed range and finite dimensional kernel. The sum of a left semi-Fredholm operator and a compact operator is again left semi-Fredholm. In the following proposition we suppose that $M$ is three-dimensional.

Proposition 3.4 For each $A \in \mathrm{L}^{3}$ the map $\mathrm{d}_{A}: \mathrm{H}^{1} \rightarrow \mathrm{L}^{2}$ is left semi-Fredholm. Moreover for any closed subspace $S$ of $\mathrm{H}^{1}$ such that $S \cap \operatorname{ker} \mathrm{d}_{A}=\{0\}$ there is $\bar{h}>0$ and $C>0$ such that for all $h<\bar{h}$ we have:

$$
\inf _{P^{\prime} \in X_{h}^{0} \otimes \mathfrak{g} \cap S} \sup _{A^{\prime} \in X_{h}^{1} \otimes \mathfrak{g}} \frac{\left|\left\langle A^{\prime}, \mathrm{d}_{A} P^{\prime}\right\rangle\right|}{\left\|A^{\prime}\right\|_{0}\left\|P^{\prime}\right\|_{1}} \geq 1 / C .
$$

- Proof: Since $\mathrm{d}^{\star} \mathrm{d}: \mathrm{H}^{1} \rightarrow \mathrm{H}^{-1}$ if Fredholm, $\mathrm{d}$ is left semi-Fredholm $\mathrm{H}^{1} \rightarrow \mathrm{L}^{2}$. Next we show that for $A$ in $\mathrm{L}^{3}$ the map $\mathrm{H}^{1} \rightarrow \mathrm{L}^{2}$ given by $P \mapsto[A, P]$ is compact. This in turn immediately implies that $\mathrm{d}_{A}$ is left semi-Fredholm.

We have a continuous (but non-compact) Sobolev injection $\mathrm{H}^{1} \rightarrow \mathrm{L}^{6}$. Moreover Hölder's inequality shows that $[\cdot, \cdot]$ is continuous $\mathrm{L}^{3} \times \mathrm{L}^{6} \rightarrow \mathrm{L}^{2}$. Thus $[\cdot, \cdot \cdot]$ is continuous $\mathrm{L}^{3} \times \mathrm{H}^{1} \rightarrow \mathrm{L}^{2}$.

Pick gauge potentials $A_{n} \in \mathrm{L}^{\infty}$ such that $\left(A_{n}\right) \rightarrow A$ in $\mathrm{L}^{3}$. Then $\left[A_{n}, \cdot\right]$ converges in norm to $[A, \cdot]$ in the space of maps $\mathrm{H}^{1} \rightarrow \mathrm{L}^{2}$. Moreover $\left[A_{n}, \cdot\right]$ is a compact map $\mathrm{H}^{1} \rightarrow \mathrm{L}^{2}$ as follows from the (Rellich) compactness of the injection $\mathrm{H}^{1} \rightarrow \mathrm{L}^{2}$. Thus $[A, \cdot]$ is a norm limit of compact operators $\mathrm{H}^{1} \rightarrow \mathrm{L}^{2}$, and hence it is compact.

Since d maps $X_{h}^{0}$ into $X_{h}^{1}$ there is trivially $C>0$ such that for all $h$ we have:

$$
\inf _{P^{\prime} \in X_{h}^{0} \otimes \mathfrak{g}, \int P^{\prime}=0} \sup _{A^{\prime} \in X_{h}^{1} \otimes \mathfrak{g}} \frac{\left|\left\langle A^{\prime}, \mathrm{d} P^{\prime}\right\rangle\right|}{\left\|A^{\prime}\right\|_{0}\left\|P^{\prime}\right\|_{1}} \geq 1 / C .
$$

Thus $\mathrm{d}_{A}=\mathrm{d}+[A, \cdot]$ is a compact perturbation of a map satisfying a (onesided) uniform discrete Inf-Sup condition (up to a finite-dimensional space). The proposition then follows from Corollaries 1.11 and 1.13 in Christiansen [8].

In geometric terms this proposition shows that the infinitesimal generators of the gauge transformations $\mathcal{G}_{Q}$ associated with Lie group valued maps $Q$ : $M \rightarrow G$ of the form:

$$
x \mapsto Q(x)=\exp (P(x)),
$$

with $P \in X_{h}^{0} \otimes \mathfrak{g}$, are far from orthogonal to $X_{h}^{1} \otimes \mathfrak{g}$. This property should be compared to the case of a commutative Lie group $G$ (e.g. Maxwell) for 
which $X_{h}^{1} \otimes \mathfrak{g}$ is stable under such gauge transformations, which, as already mentioned, provides an interpretation of the conservation property (3.9) in terms of Noether's theorem.

Typically one would like to apply the Babuska-Brezzi compatibility property along trajectories $t \mapsto A(t) \in X_{h}^{1} \otimes \mathfrak{g}$. For this purpose we mention that Corollary 1.17 in Christiansen [8] automatically extends certain estimates which follow from Proposition 3.4 to hold uniformly for sets of gauge potentials $A$ that are compact in $\mathrm{L}^{3}$.

\section{Perspectives}

We believe that Yang-Mills equations provide a testbench for many problems in the theory of discretizations of constrained PDEs and we mention in this conclusion two directions for future investigations.

Multiplying the Lie bracket on $\mathfrak{g}$ by a real parameter provides some freedom in tuning the strength of the non-linearity. That is, we can introduce a coupling parameter $\gamma \in \mathbb{R}$ and consider the curvature:

$$
\mathcal{C}_{\gamma}(A)=\mathrm{d} A+(\gamma / 2)[A, A] .
$$

We are interested in trajectories obtained from the associated Lagrangian:

$$
\mathcal{L}_{\gamma}\left(A, A^{\prime}\right)=(1 / 2)\left\|A^{\prime}\right\|^{2}-(1 / 2)\left\|\mathcal{C}_{\gamma}(A)\right\|^{2} .
$$

The limit $\gamma \rightarrow 0$ is often referred to as the weak coupling limit, while the case $\gamma=0$ is equivalent so Maxwell's equations. Various series expansions e.g. in powers of $\gamma$, around the "trivial" case $\gamma=0$ are used in many circumstances (for instance KAM theory) to provide information where other methods fail.

We mention also that, in physics, quantized versions of the Yang-Mills equations are the most important. Given a representation of the Lie group $G$ on a vector space $V$, a gauge potential on $M$ gives rise to a connection on the space of $V$-valued maps on $M$ which is compatible with $G$, and vice versa. The lattice gauge theory due to Wilson consists in using a cubic lattice and attach Lie group elements to each edge, representing the parallel transport of a connection. The curvature of the connection has degrees of freedom attached to each square of the lattice, called Wilson loops. This discretization appears to be linked to the presently proposed finite element method in much the same way as, for Maxwell's equations, the Yee scheme, which is a staggered finite difference scheme on cubic lattices, is related to variational mixed finite element methods.

\section{Acknowledgements}

This work was inspired by a discussion between Douglas N. Arnold, Alan D. Rendall and the second author at an IMA "hot topics" workshop on Numerical Relativity in June 2002. We thank Geir Ellingsrud and Roman Kozlov for interesting discussions on connections and geometric integration. We also thank Per Christian Moan and Jan B. Thomassen for remarks on which $§ 4$ is partly based. 


\section{References}

[1] D.N. ARnold: Differential complexes and numerical stability; Plenary address delivered at ICM 2002, International Congress of Mathematicians, Beijing 2002.

[2] R. BERndT: An introduction to symplectic geometry; AMS Graduate Studies in Mathematics, Vol. 26, 2000.

[3] D. Boffi, P. Fernandes, L. Gastaldi, I. Perugia: Computational models of electromagnetic resonators: analysis of edge element approximation; SIAM J. Numer. Anal., Vol. 36, No. 4, p. 1264 - 1290, 1999.

[4] D. Boffi: A note on the DeRham complex and a discrete compactness property; Appl. Math. Lett., Vol. 14, No. 1, p. 33 - 38, 2001.

[5] A. Bossavit: Mixed finite elements and the complex of Whitney forms; The mathematics of finite elements and applications, VI (Uxbridge, 1987), p. 137 - 144, Academic Press, London, 1988.

[6] F. BREzzI: On the existence, uniqueness and approximation of saddlepoint problems arising from Lagrangian multipliers; RAIRO Anal. Numér., Vol. 8, No. R-2, p. 129 - 151, 1974.

[7] F. Brezzi, M. Fortin: Mixed and hybrid finite element methods; Springer-Verlag, 1991.

[8] S. H. Christiansen: Discrete Fredholm properties and convergence estimates for the Electric Field Integral Equation; Math. Comp., Vol. 73, No. 245 , p. $143-167,2004$.

[9] S. H. Christiansen: A div-curl lemma for edge elements; SIAM J. Numer. Anal., accepted.

[10] P. Ciarlet JR., J. Zou: Fully discrete finite element approaches for time-dependent Maxwell's equations; Numer. Math., Vol. 82, p. 193 - 219, 1999.

[11] L. Demkowicz: hp-adaptive finite elements for time-harmonic Maxwell equations; Topics in computational wave propagation, p. 163 - 199, Lect. Notes Comput. Sci. Eng., Vol. 31, Springer, Berlin, 2003.

[12] S. K. Donaldson, P. B. Kronheimer: The geometry of four-manifolds; Oxford University Press, 1990.

[13] J. J. Duistermant, J. A. C. Kolk: Lie groups; Springer, 2000.

[14] D. Eardley, V. Moncrief: The global existence of Yang-Mills-Higgs fields in $\mathrm{M}^{3+1}$; Comm. Math. Phys., Vol. 83, p. $171-212,1982$.

[15] G. B. Folland: Real Analysis: Modern techniques and their applications, Second Edition; John Wiley \& Sons, 1999.

[16] S. I. Gelfand, Y. I. Manin: Homological algebra; Springer-Verlag, Berlin, 1999. 
[17] V. Girault, P.-A. Raviart: Finite element methods for Navier-Stokes equations; Springer-Verlag, Berlin, 1986.

[18] E. Hairer, C. Lubich, G. Wanner: Geometric numerical integration: structure preserving algorithms for ordinary differential equations; Springer Series in Computational Mathematics, No. 31, Springer, 2002.

[19] R. Hiptmair: Finite elements in computational electromagnetism; Acta Numerica, p. 237 - 339, Cambridge University Press, 2002.

[20] P. Joly: Variational methods for time-dependent wave propagation problems; Topics in computational wave propagation, p. 201-264, Lect. Notes Comput. Sci. Eng., 31, Springer, Berlin, 2003..

[21] F. KikUCHI: On a discrete compactness property for the Nédélec finite elements; J. Fac. Sci. Univ. Tokyo, Sect. 1A Math., Vol. 36, p. $479-490$, 1989.

[22] S. Klainerman, M. Machedon: Finite energy solutions for Yang-Mills equations in $\mathbb{R}^{3+1}$; Ann. of Math. (2), Vol. 142, No. 2, p. $39-119,1995$.

[23] S. Lang: Differential and Riemannian manifolds, Third edition; SpringerVerlag, New York, 1995.

[24] L. Lehner: Numerical relativity: a review; Class. Quantum Grav., Vol. 18 , p. $25-86,2001$.

[25] B. Leimkuhler and S. Reich: Simulating Hamiltonian dynamics; Oxford University Press ???, 2004.

[26] J. E. Marsden, M. West: Discrete mechanics and variational integrators; Acta Numerica, p. 357 - 514, Cambridge University Press, 2001.

[27] P. Monk: Finite element methods for Maxwell's equations; Oxford University Press, 2003.

[28] J.-C. NÉDÉLEC: Mixed finite elements in $\mathbb{R}^{3}$; Numer. Math., Vol. 35, p. $315-341,1980$.

[29] J.-C. NÉDÉLEC: A new family of mixed finite elements in $\mathbb{R}^{3}$; Numer. Math., Vol. 50, No. 1, p. $57-81,1986$.

[30] J.E. Roberts, J.-M. Thomas: Mixed and hybrid methods; p. 523-640, in P.G. Ciarlet, J.-L. Lions (eds.), "Handbook of numerical analysis, Vol. II, Finite element methods (Part 1)", North-Holland, 1991.

[31] R.A. RYAN: Introduction to tensor products of Banach spaces; Springer Monographs in Mathematics, Springer, 2002.

[32] I. Segal: The Cauchy problem for the Yang-Mills equations; J. Func. Anal., Vol. 33, No. 2, p. 175 - 194, 1979.

[33] R. Sorkin: The electromagnetic field on a simplicial net; J. Math. Phys., Vol. 16, No. 12, p. 2432 - 2440, 1975. 
[34] T. TAO: Local well-posedness of the Yang-Mills equation in the temporal gauge below the energy norm; J. Differential Equations, Vol. 189, No. 2, p. $366-382,2003$.

[35] M. TAYlor: Partial differential equations, Vol. II Qualitative studies of Linear equations; Springer-Verlag, New York, 1996.

[36] K. Uhlenbeck: Connections with $\mathrm{L}^{p}$ bounds on curvature; Comm. Math. Phys., Vol. 82, No. 1, p. $31-42,1982$.

[37] R. M. WALD: General relativity; University of Chicago Press, 1984.

[38] A. WeIL: Sur les théorèmes de de Rham; Comment. Math. Helv., Vol. 26, p. $119-145,1952$.

[39] H. Whitney: Geometric integration theory; Princeton University Press, 1957. 